\title{
Binocular foveation in reading
}

\author{
Richard Shillcock, Matthew Roberts, Hamutal Kreiner, and Mateo Obregón \\ University of Edinburgh, Edinburgh, Scotland
}

\begin{abstract}
We present a theory of foveation in normal binocular reading. We consider the pervasive, nontrivial binocular fixation disparities (FDs) observed in reading and relate them to the computational problem of resolving retinal disparities in depth perception. We infer that the right eye's fixation being to the right of the left eye's in reading promotes binocular fusion in challenging conditions. We then show a different (nonfusional) processing advantage for the right eye's fixation being to the left of the left eye's in reading conditions in which binocular fusion is assured, by modeling the combined influence of foveal splitting, contralateral preference, ocular prevalence, and fixation disparity. This synthesis of anatomically grounded research in different aspects of visual processing produces a theory of foveation in reading that matches current data and makes testable predictions.
\end{abstract}

Our goal is to present a theory of foveation in binocular reading, explaining the variability in the positioning of the eyes to project the text across the two foveas. During normal reading, the left and right eyes frequently fixate different points in the line of text. The left eye may fixate to the left of the right eye, which is termed an uncrossed fixation disparity (FD), or to the right, termed a crossed FD. Several studies have suggested a pattern of predominantly conjoint or uncrossed FDs in reading (Blythe et al., 2006; Juhasz, Liversedge, White, \& Rayner, 2006; Liversedge, White, Findlay, \& Rayner, 2006), but two recent reports have revealed a substantial departure from this pattern (Nuthmann \& Kliegl, 2009; Roberts, Shillcock, Kreiner, \& Obregón, 2010). We present a theory of binocular foveation in reading grounded in research on stereopsis and on the structure of the visual pathways between the fovea and the cortex. The theory has three central premises: that individuals unconsciously manipulate their FD in response to viewing conditions to facilitate fusion, that uncrossed FDs facilitate binocular fusion more than crossed FDs do, and that crossed FDs facilitate postfusional processing more than uncrossed FDs do. These tenets lead to the conclusions that, when reading conditions militate against binocular fusion, readers tend to opt for uncrossed FDs and that, when binocular fusion is unproblematic, readers tend to opt for crossed FDs.

We begin with a detailed description of the variations in FD in reading. We then consider theoretical explanations of disconjugacy ${ }^{1}$ during saccades (which results in FD) and conclude that some additional explanation is required to account for the range of FDs reported between reading studies. Our explanation proceeds from the observation that the computational consequences of FD resemble the correspondence problem encountered in depth perception: Both involve a directional noncorrespondence between the images on the respective retinas, mediated by position with respect to the horopter. From our review of the stereopsis literature, we conclude that binocular fusion in reading is more easily achieved during uncrossed FDs than during crossed FDs and that viewing conditions such as luminance, blur, font size, and viewing distance prompt changes in FD.

We complete our explanation by providing a rationale for the large number of crossed FDs reported by Nuthmann and Kliegl (2009) and by Roberts et al. (2010). We describe a high-level model of the visual pathways from fovea to cortex. The model shows that the combination of known aspects of the visual system, such as ocular prevalence and bias for contralateral over ipsilateral projection, result in a robust processing advantage for crossed FDs. This finding leads to our overall conclusion that crossed FDs are advantageous for readers when fusion is unproblematic.

\section{Fixation Disparity}

FD in reading. Binocular studies of reading, both of children and adults, date back many decades but have become a recent focus of research due to the greater availability of binocular eyetrackers (Bassou, Granié, Pugh, \& Morucci, 1992; Blythe et al., 2006; Clark, 1935; Heller \& Radach, 1999; Hendriks, 1992, 1996; Hendriks, Kolk, \& van der Wildt, 1991; Juhasz et al., 2006; Kliegl, Grabner, Rolfs, \& Engbert, 2004; Liversedge et al., 2006; Nuthmann \& Kliegl, 2009; Roberts et al., 2010; Schmidt, 1917; Taylor, 1966; Ygge \& Jacobson, 1994).

The data on direction of FD vary substantially across recent studies, regarding the relative proportions of crossed, uncrossed, and conjoint fixations (see Table 1); this variability is the primary explicandum for a theory of binocular foveation. In addition to the data in Table 1, Yen and Radach (2007) reported more uncrossed than crossed FDs, but no precise numbers; Heller and Radach (1999) did not state the direction of the FDs. Liversedge et al. (2006) stated

R.Shillcock, rcs@inf.ed.ac.uk 
Table 1

Percentages of Different Types of Fixation Disparity From Recent Studies

\begin{tabular}{lccc}
\hline \multicolumn{1}{c}{ Study } & Conjoint & Crossed & Uncrossed \\
\hline Liversedge et al. (2006) & 53 & 8 & 39 \\
Juhasz et al. (2006) & 55 & 18 & 27 \\
Blythe et al. (2006) (adult data) & 48 & 12 & 40 \\
$\quad " \quad$ " (child data) & 39 & 24 & 37 \\
Nuthmann \& Kliegl (2009) & 58 & 39 & 3 \\
Roberts et al. (2010) & 14.5 & 85 & 0.5 \\
\hline
\end{tabular}

"At present, it is not clear why this difference [between crossed and uncrossed] between the present data and those of Kliegl et al. [2006] occurred. Clearly, further research is required to more fully understand this interesting difference" (note 6, p. 2368; see also Kirkby, Webster, Blythe, \& Liversedge, 2008, p. 747). Nuthmann and Kliegl (2009) concluded that "the marked differences across studies suggest that not only individual differences and developmental aspects but also factors related to the experimental setting determine whether the adopted fixation disparity is predominantly crossed or uncrossed" (p. 22) and listed several candidate explanations: eyetracker technology, calibration procedure, ${ }^{2}$ saccade detection method, viewing distance, visual angle per letter, head movement constraint, color of text and background, room illumination, and language used. We may add to this list the issue of sentential versus paragraph stimulus materials. ${ }^{3}$
The data cited in Table 1 were acquired in different experimental conditions that are relevant to understanding FD in reading. Figure 1 graphs the variation in FD in reading studies and demonstrates the relation between the proportions of the three fixation types (crossed, uncrossed, conjoint) and the size of the angle subtended by a single character on the retina. ${ }^{4}$ There are only substantial proportions of uncrossed FDs in the studies in which the font subtends a smaller angle on the retina; coincidentally, these are the studies using a dual Purkinje display, in which bright letters are presented on a black background in a darkened room. In contrast, the studies showing more substantial proportions of crossed FDs use dark text on light backgrounds in normal lighting conditions with larger text.

Liversedge et al. (2006, Figure 2) showed the proportions of uncrossed FDs and conjoint fixations in approximately complementary distribution across the line of text. Nuthmann and Kliegl (2009) reported a complementarity in the proportions of conjoint fixations and crossed FDs. Thus, different data sets tend to contain complementary proportions of conjoint fixations and one directional FD, with the other direction of FD relatively marginalized. We have analyzed this situation in Figure 2 by taking the estimated mean FD of each data set (it has to be estimated, because FDs of less than one character are standardly taken to be conjoint) and by situating each mean along the $x$-axis. Each vertical line represents one of the data sets in Table 1, identified by the initial of the first author of the

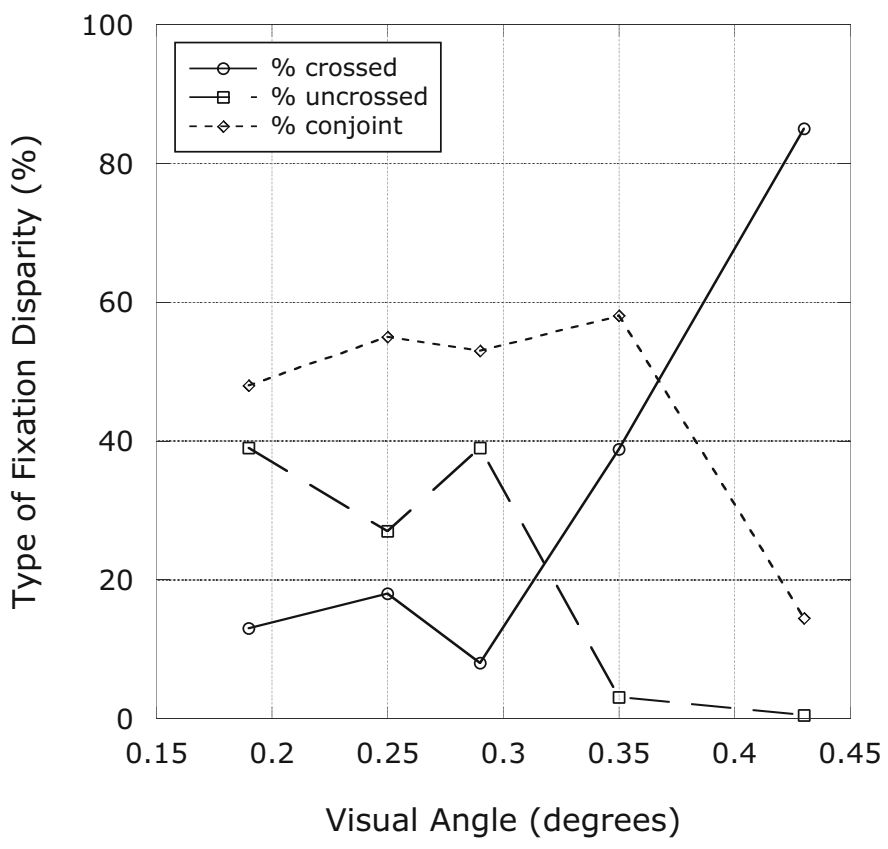

Figure 1. Meta-study of published data comparing percentages of crossed, uncrossed, and conjoint FDs with retinal angle subtended by a single monospaced letter. From smallest visual angle on the left, data sets are from Blythe et al. (2006), Juhasz et al. (2006), Liversedge et al. (2006), and Nuthmann and Kliegl (2009), and from a subset of 14 from our present English text-reading corpus data. Note that there are procedural differences between the five studies (see text for details); one such difference is that the Nuthmann and Kliegl data were acquired using binocular calibration. 


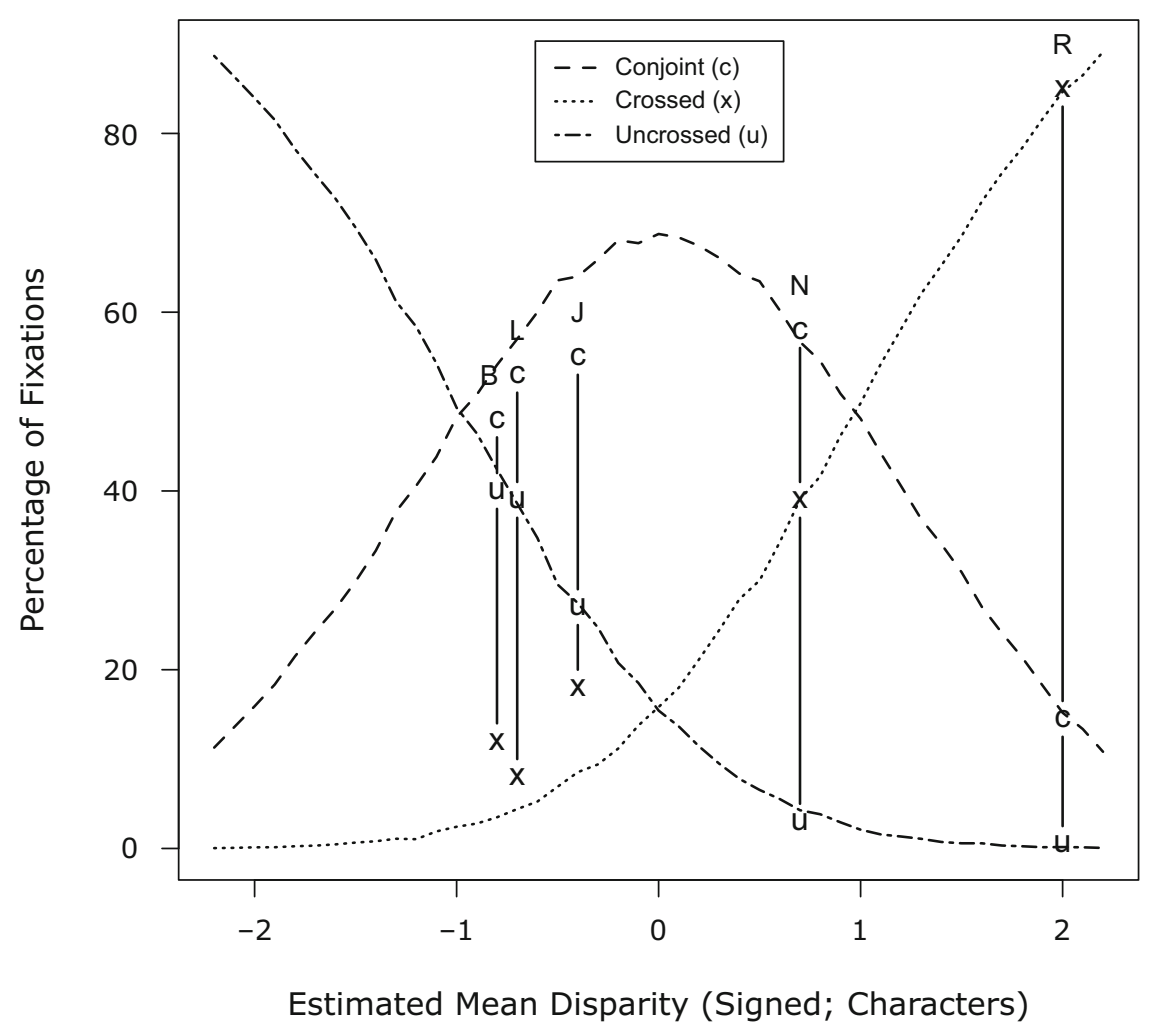

Figure 2. Proportions of uncrossed, conjoint, and crossed fixation disparity (FD) for estimated overall mean disparities greater than -2 and less than 2 and assumed $S D s$ of 1 , where $F D$ is $\mathbf{L}-\mathbf{R}$, in characters. Vertical lines show the best-fitting means for the proportions reported in the studies summarized in Table 1 ; the initials $B, L, J, N$, and $R$ refer to the particular data sets. The estimated means themselves are less important than is the demonstration that the varying percentages of the fixation subtypes (conjoint, crossed, uncrossed) in the reported data sets may be seen as the outcome of partially overlapping distributions of those subtypes, yielding different mean FDs overall for those data sets. The mean FD overall for a data set, and its sign, must then be explained. See the text for this explanation.

respective article. The differing proportions of disparity subtypes within each data set may be seen as the intersection of partially overlapping distributions of the three subtypes. Thus, change in the mean FD appears as complementary variation between the dominant FD (crossed or uncrossed within a particular data set) and conjointness, with the nondominant FD appearing relatively unaffected; for instance, the Nuthmann and Kliegl data ("N" in Figure 2) have an estimated overall FD of less than one character and are composed of 58\% conjoint fixations and $39 \%$ crossed fixations, so that any small variation about the overall mean FD results in larger complementary variation between the conjoint and crossed categories and in smaller variation in the percentage of uncrossed fixations. In the rest of the present article, we address the question of why any FD happens and why it occurs in one or other direction (crossed or uncrossed). Figure 2 demonstrates that the reported data sets can all coexist as accurate depictions of binocular fixation behavior within orderly relations between the equally legitimate conjoint, crossed, and uncrossed subtypes.

In summary, FD is pervasive in reading, but is variable, with the basic data on the direction of the FD and its role in reading a central research question. Below, we will pursue the hypothesis that these data sets are mutually compatible; they do not reflect error in the system, but are strategic responses to the stimulus and the task. We begin with a review of the literature on FD in nonreading tasks.

FD in nonreading tasks. Several nonreading studies have shown substantial disconjugacy in binocular saccades along with FD. Enright (1998) reported an experiment in which binocular recordings were made of 3 participants' repeated saccades between two points ( 20 or $26 \mathrm{~cm}$ away), requiring version, vergence, and isovergence movements. The chief finding was of a binocularly unbalanced saccade that resulted in good foveation of the target by one or other eye, with substantial deviation of the partner eye, which then required an asymmetric vergence movement. This behavior still occurred in the isovergence case (a task more comparable, in terms of depth, to reading), although the deviation of the initially less accurate eye was smaller (see Enright, 1998, Figure 10). There were no clear conclusions regarding direction and eccentricity of the target point from each eye. Cornell, Macdougall, Predebon, and Curthoys (2003) reported data from a task requiring symmetrical vergence movements in natural conditions. 
Participants produced significant underconvergence for near fixations $(52.5,30$, and $21 \mathrm{~cm}$, comparable with reading distances) and overconvergence for far fixations. For the near fixations, $85 \%$ of errors were from -30 to +120 arc min. Comparing the situation in these vergence tasks with the crossed/uncrossed FDs in reading, underconvergence produces an uncrossed FD and overconvergence produces a crossed FD. ${ }^{5}$ We conclude from these two studies that binocular FD - as opposed to precisely conjoint fixations - often happens in three-dimensional nonreading tasks without incurring diplopia; Cornell et al. remarked specifically on the absence of diplopia.

It is worth noting that, in real-world viewing, the great majority of objects do not fall on the horopter, the circle on which the fixation point sits. For instance, when we fixate an object on a crowded desk, there are typically objects both in front of and beyond the horopter; such objects are not, therefore, projected to corresponding points on the two retinas, but the relevant images still need to be coordinated (by binocular fusion or suppression). Saccades requiring a vergence change are more representative of real-world viewing than are the persistent near-isovergent saccades required in reading.

In other studies of binocular coordination in nonreading tasks, the saccades have been exclusively isovergent; these tasks are, therefore, more comparable with reading. Collewijn, Erkelens, and Steinman (1988) reported using a scleral sensor coil, in which 3 participants repeatedly fixated between two isovergent LEDs in a dimly lit room. They reported a "binocular fixation error of about $0.3^{\circ}$ in the divergent direction" (p. 168; i.e., uncrossed FDs, which formed the majority). They also reported the typical undershooting seen previously in the literature. Fioravanti, Inchingolo, Pensiero, and Spanio (1995) used an infrared limbus-tracking system to study binocular saccades within an isovergent circle of LEDs and found uncrossed FDs for adults and for 11- to 13-year-old children but found crossed FDs for 5- to 10-year-old children. Yang and Kapoula (2003) used an oculometer (Bach, Bouis, \& Fischer, 1983) to study saccades made to LEDs in near and far isovergent circles by children and adults and found substantial FDs in the uncrossed direction (Figure 1, p. 556), with greater disconjugacy in children and for near targets. Other articles have suggested the same conclusion; for instance, Cornell et al. (2003) cited Malinov, Epelboim, Herst, and Steinman (2000), calculating vergence errors ranging from $1.5^{\circ}$ to $3^{\circ}$. (See also Collewijn, Erkelens, \& Steinman, 1995, and Erkelens, Steinman, \& Collewijn, 1989 , who reported errors of up to $6^{\circ}$.)

The first conclusion from these binocular-nonreading studies is that immediate or sustained, precise conjoint foveation does not always happen and is not always necessary; "saccades are not temporally and spatially conjugate as has often been assumed" (Kirkby et al., 2008). No diplopia was reported in the studies reported above, although Yang and Kapoula (2003) said "coordination is particularly poor at near [distances] and could compromise single binocular vision" (p. 554). Cornell et al. (2003) argued that individuals are tolerant of physiological diplopia in normal, free-viewing situations in which there is head movement and multiple fixations in a complex visual environment. Binocular fusion is robust when there are large objects in the visual field (Schor \& Wood, 1983; Tyler, 2004, p. 21), and such stimuli, once fused, are resistant to diplopia. These data contrast with the very strict conditions on binocular fusion, as in the classic statement of Panum's fusional area ( 15 arc min), found when demanding experimental conditions are imposed (e.g., no head movement and the brief, single presentation of impoverished stimuli). The second conclusion from these studies is that uncrossed (divergent) FDs are widely found in nonreading tasks.

What explanations have been offered for disconjugacy in saccades and for binocular FD? Yang and Kapoula (2003) evaluated two theoretical possibilities: that they may result from unequal cortical signals to each eye or from asymmetry in the oculomotor musculature. They drew two principal conclusions: First, even if, as required by Hering's law of equal innervation, a single signal is generated by the cortex to move the eyes, it is clear that the signal has diverged along different pathways by the time it reaches the eyes. This process of fine tuning is less evident in children and must be learned: "Learning and adaptation are needed for the fine tuning of motor commands for each eye to enable normal quality of binocular coordination in adults" (p. 559). Although such fine tuning might appear to be strong evidence against Hering's law, they acknowledge the possibility that "it is basically true but imperfect" (Ibid.). Second, they show that the hypothesis that saccadic disconjugacy is caused by muscular asymmetry is incompatible, both with their own behavioral data and with the known facts of physiological development. This hypothesis is based on the fact that the lateral rectus muscle (controlling abduction, i.e., away from the nose) produces more acceleration in the saccade than does the medial rectus muscle (controlling adduction, i.e., toward the nose), so that the two eyes diverge. They concluded that "the distance-dependent disconjugacy of the amplitude of saccades is most likely due to immature cortical or subcortical control of saccade signals when the eyes are converged than to muscular difference between the two eyes" (p. 560).

The variation in FD that has been reported in different studies of reading supports the conclusion that muscular asymmetry is not its primary cause. Muscular asymmetry (specifically, stronger external recti) would seem consonant with a pattern of largely uncrossed FDs but not with the opposite pattern reported by Nuthmann and Kliegl (2009). The hypothesis presented here builds on Yang and Kapoula's (2003) assertion that "the binocular coordination of saccades is not built-in, but is a process developing through visual experience and learning" (p. 560). This claim implies that experienced readers may exercise a degree of independent control over their two eyes. We posit that they may use that control to optimize their FD for the reading conditions at hand. The flexibility of the system is apparent in data from a heterogeneous group of amblyopic adults reported by Conner, Odom, Schwartz, and Mendola (2007), who suggested that radical pathological binocular FD may lead to the system suppressing some fine-grained 
central input, while maintaining processing in peripheral vision. Exactly how the adaptiveness that we propose for binocular FD is realized is a matter for further research.

In conclusion, noncorrespondence in much of the image is unavoidable in the three-dimensional world: Substantial FDs have been widely observed in a range of viewing tasks, and we should expect them in reading, with no noticeable diplopia. The direction of the FD is frequently uncrossed, but no principled explanation of the direction of the FD has emerged beyond those based on error and hypometric inertia (see, e.g., Cornell et al., 2003). We now show how FDs in reading entail some of the same computational issues identified in depth perception.

\section{FD, Retinal Disparities (RDs), and Depth Perception}

Depth perception enables us to negotiate the visual world and perceive the solidity of objects. There is a critical difference between perceiving objects behind and in front of the horizontal horopter (on which all points, including the binocular fixation point, produce single vision and project to corresponding points on the retinas), all in a single binocular perception of the world. A conjointly fixated point is projected onto the fovea of the left and right eyes. When projected onto the retina, objects beyond the horopter give rise to uncrossed RDs (defined below). Objects in front of that plane of focus give rise to crossed RDs. This difference is a qualitative one in terms of the sign of the relative warping of the images on the two retinas with respect to the relevant corresponding points (see, e.g., Hershenson, 1999, p. 36, for the textbook description of both types of RD; cf. Blakemore, 1969; Collewijn, Steinman, Erkelens, \& Regan, 1991).
Thus, if the viewer raises his or her finger in front of a distant tree, fixating on the finger produces an uncrossed disparity for the tree; the left eye (LE) sees tree-finger (from left to right across the field), the right eye (RE) sees finger-tree, and overlaying the two monocular images produces an uncrossed double image of the tree: treefinger-tree. This case is "uncrossed," because the tree is to the left in the LE and to the right in the RE. In the crossed case, the viewer fixates on the tree, the LE sees tree-finger, and the RE sees finger-tree, which produces the crossed double image finger-tree-finger. This case is "crossed," because the finger is to the right in the LE and to the left in the RE. This is the definition of relative disparity, in which an object off the horopter is seen in depth relative to the horopter and the viewer.

Figure 3 illustrates how a crossed FD generates the signed directional consequences of an uncrossed RD. Figure $3 \mathrm{~A}$ shows that in an uncrossed FD in reading, the $o$ in fox in the LE occurs to the right of the $o$ in $f o x$ in the RE (equivalent to a crossed RD). Conversely, in Figure 3B, in a crossed FD, the $o$ in fox in the LE occurs to the left of the $o$ in $f o x$ in the RE (an uncrossed RD).

In the (recent, small) binocular reading literature, the term $F D$ is used to refer to the distance between the two fixation points on the text. We have referred above, to the (older, larger) stereopsis literature, in which an RD describes the relative placement of images on the two retinas. ${ }^{6}$ There is an unfortunate use of the same terms "crossed" and "uncrossed" in the binocular-reading and stereopsis literatures, respectively, to refer to the relationship between the two eyes' fixation points on the text and to directional noncorrespondences between the images on the retinas; in making an argument relevant to both
A

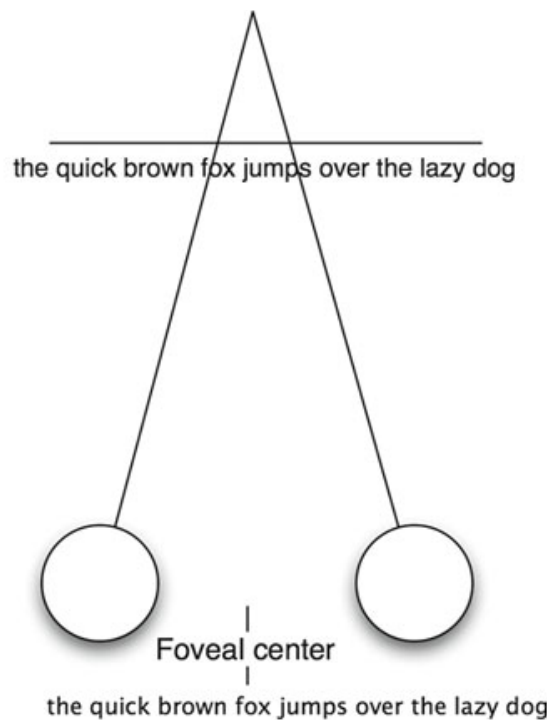

Left eye

Right eye the quick brown fox jumps over the lazy dog
B

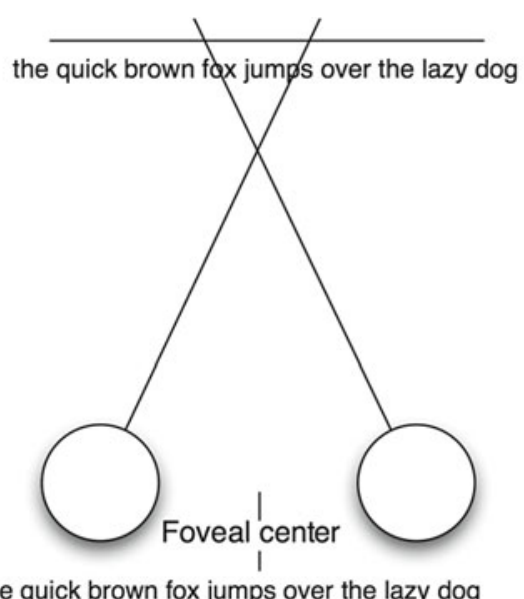

Left eye

the quick brown fox jumps over the lazy dog

Right eye the quick brown fox jumps over the lazy dog

Figure 3. Fixation disparity (FD) produces noncorresponding images at the two retinas. (A) An uncrossed FD in reading, equivalent to a crossed retinal disparity (RD). (B) A crossed FD, equivalent to an uncrossed RD. The disparity $X$ between the two images is signed, + or - , and is analogous to that observed in depth perception for objects not on the horopter. The uncrossed FD effectively brings the text in front of the horopter, and the crossed FD sends it behind the horopter (as defined by the point of intersection of the two lines of sight). The sign has implications for the processing domain that achieves binocular fusion. See the text for details. 
literatures, we are explicit throughout in distinguishing FDs from RDs. (This distinction naturally applies across viewing tasks other than reading.)

Thus, the binocular-reading research community understand a "crossed disparity" to refer to the right eye's fixation point on the text being to the left of the left eye's fixation point, and they have not been concerned with the precise implications for the location of the images on the retina. In contrast, the rest of the vision-science community understands the older usage of "crossed disparity" as referring to the difference between the images on the retinas associated with a midline object situated between the viewer and the intersection of the lines of sight from the two eyes (see above); the vision science community has typically been less interested in situations in which the two eyes do not, in principle, fixate the same point in the world and has usually seen this as "vergence error." In the present article, we have tried to explore the relation between these two situations in terms accessible to both research communities. For future reference, crossed fixation points on the page $\equiv$ uncrossed disparities on the retina $\equiv$ over convergence $\equiv$ esodisparity and uncrossed fixation points on the page $\equiv$ crossed disparities on the retina $\equiv$ underconvergence $\equiv$ exodisparity.

To achieve binocular perception, the cortex needs to perform qualitatively different coordinations of the relevant parts of the images on the retinas in crossed and uncrossed RDs. It should also be clear that fusion is a more complex computation than simple geometric translation. Most real-world scenes require a constant interplay between the processing of crossed and uncrossed RDs as objects are fixated in near and far space, and a single binocular percept is created of the whole visual field; these opposite RDs are defined with respect to each other and are typically copresent. ${ }^{7}$ However, it is shown below that different processing considerations attach to the domain in front of the horopter and to the domain behind it.

Normally, we formalize depth perception as a situation in which we fixate conjointly on one object and in which a second object, either in front of or behind the horopter, is seen in depth; the second object creates a relative disparity at the two retinas. The problem for the visual system is the central one of coordinating the two noncorresponding whole retinal images. The horopter, created by the current fixation point, is essential to understanding depth processing: Regions off the horopter are perceived as having depth with respect to the horopter and the viewer. The relative disparity of an object off the horopter is quantified as the difference between its absolute disparity and the absolute disparity of the fixation on the horopter (where absolute disparity is the angle subtended at the object by the lines of sight from the two eyes). Note, first, that there does not need to be an actual object fixated on the horopter for absolute disparity to obtain for objects off of the horopter (and, indeed, the two lines of sight may not actually cross if there is vertical disparity; Collewijn et al., 1991, p. 122). Note, second, that, although absolute disparity (the angle between the two lines of sight) provides little information for depth perception in the presence of other cues, absolute disparity information is always available, as is evidenced by the central ability of the eyes to converge on an isolated stimulus (cf. Howard \& Rogers, 2002, pp. 151-152); indeed binocular neurons in (macaque) V1 select for absolute disparity and not for relative disparity (Cumming \& Parker, 1999).

Consider the following range of circumstances: When there are two objects at different distances from the horopter, their relative disparities may be defined with respect to each other and independent of ocular vergence and conjugate eye movements, or they may be defined with respect to the horopter. When there is a single object off the horopter, its relative disparity may be calculated with respect to a fixated object (on the horopter); we will see, below, that it is critical whether the object is in front of or beyond the horopter. When there is a single fixation plane, with no other point of reference, there is only an absolute disparity, with little or no depth perception generated on the basis of this absolute disparity. Finally, when there is an FD (the two eyes not fixating conjointly), such as in our reading case, and substantially a single fixation plane with no other point of reference, we still have an absolute disparity, but a virtual fixation point in which the lines of sight intersect in front of or behind the text. The two eyes receive overall disparate (Howard \& Rogers, 1995, p. 243) images of the text which must still be coordinated. We have seen that this overall disparity is signed (the crossed or uncrossed cases are qualitatively and directionally different). Although this overall disparity does not reveal anything about the structure of the visual scene (indeed, in the reading case, the text is substantially all at the same depth), we can still generalize from the other cases of the coordination of disparate images. Note, again, that the coordination of the two images is always a more complex task than simple geometric translation: In every fixation, the full panoply of depth mechanisms and processes is available. ${ }^{8}$ As demonstrated above, the central factor in the range of cases of depth perception considered is the location of the horopter; we will review, below, the evidence for the critical signed distinction between object location in front of and beyond the horopter.

The central point we make here is that the computational consequences of depth perception generalize to the processing of FD in reading. The visual system is required to coordinate noncorresponding images on the two retinas, with a critical signed difference in direction. In an uncrossed FD, the principal visual axes from the reader's eyes meet behind the page or screen; in the crossed case they meet in front.

The essence of FDs in reading is that an uncrossed FD places the text within the horopter and that a crossed FD places it beyond the horopter (respectively defined by the virtual and real crossing of the lines of sight). This formal generalization from stereopsis to reading means that the processing of FD in reading inherits the processing distinctions reported in stereopsis regarding depth relations in front of and beyond the horopter.

Studies of depth perception suggest that the brain solves the two computational problems posed by positive and negative RDs relatively independently. The initial case was made by Richards (1970, 1971a, 1971b), 
who presented psychophysical and clinical evidence of separate detectors for crossed and uncrossed RDs. Later research supports this claim (Birch \& Foley, 1979; DeAngelis, 2000; Foley, 1976; Foley \& Richards, 1978; Poggio \& Fischer, 1977; Regan \& Spekreijse, 1970). Below, we review studies comparing crossed with uncrossed RDs, further supporting the conclusion that there are at least ${ }^{9}$ two anatomically and functionally dissociable processing domains corresponding to the two types of RD. ${ }^{10}$

In summary, the computational problem faced by the reader of coordinating noncorresponding retinal images resembles the problem of perceiving objects in the real world in depth. In depth perception, the two aspects of the problem are solved in different (crossed and uncrossed $\mathrm{RD}$ ) processing domains, although these processing domains are intimately connected and complementary aspects of depth perception. However, the relationship between crossed and uncrossed RDs is not symmetric, as we see below when we review a substantial literature showing that crossed RDs offer a number of general advantages in nonreading tasks. Further below, we explore how different viewing conditions may offer differential advantages to crossed and uncrossed FDs in reading.

\section{Crossed RDs Are Prioritized in Nonreading Tasks}

Mustillo (1985) discussed evidence from studies in a range of paradigms - psychophysics, eye movements, human development, clinical cases, human electrophysiological data, animal behavior, and neurophysiology — and concluded that crossed RDs are processed more readily and more efficiently than are uncrossed RDs. We update Mustillo's review and develop the relevant parts toward the issue of FDs in reading.

Stereofusion. There is a clear advantage of crossed RDs in recruiting visual information for fusion. Dengler and Kommerell (1993) investigated the limits of RD in a task requiring participants to report depth perception in relation to a pair of $3^{\circ}$ discs presented, respectively, to the fovea of one eye and the periphery of the other. They showed that crossed RDs could be processed at larger angles of separation than could uncrossed RDs. Woo and Sillanpaa (1979) also reported that the threshold for stereopsis was lower in crossed than in uncrossed RDs. This difference reflects the real-world constraint that an object's images in the two eyes are more disparate when the object is closer rather than when it is farther away. This finding replicates the earlier one of Richards and Foley (1971), who showed some participants being able to process as much as $16^{\circ}$ of a crossed RD (up to four times greater than uncrossed RDs; see also Grabowska, 1983; Lasley, Kivlin, Rich, \& Flynn, 1982; Schor \& Wood, 1983). These data speak directly to the flexibility of the visual system in maintaining fusion in relatively natural scenes, despite large RDs.

There is also a temporal dimension to stereofusion. When a lag is introduced between the presentation of the stimuli to each eye, depth is perceived more robustly during crossed RDs than during uncrossed RDs (Godek \& Lawson, 1973; Ogle, 1963). Similarly, when RD information is only briefly available, participants process crossed RDs at shorter presentations than they do uncrossed RDs (Beverley \& Regan, 1974; Regan \& Beverley, 1973a, 1973b).

In summary, the greater effective range of stereofusion in crossed RDs (i.e., uncrossed FDs) implies that uncrossed FDs are more effectively and efficiently fused (although the observed data from reading would rarely seem to approach the limits of fusion reported by Richards \& Foley, 1971).

Stereoacuity. Stereoacuity has been generally reported as being higher in crossed RDs than in uncrossed RDs in depth perception tasks (e.g., Lam, Tse, Choy, \& Chung, 2002), although some researchers have failed to find a difference (see Howard \& Rogers, 1995, p. 166, for a review). When fine distinctions are required in stereofusion, uncrossed FDs may, therefore, be more appropriate.

$R D$ and the visual field. Richards and Regan (1973) showed that there are different stereopsis field maps for crossed and uncrossed RDs, with the former being larger than the latter, extending both above and below the fovea, and being substantially biased toward the right visual field (RVF); the field map for the uncrossed RDs is relatively small (and is also biased toward the RVF).

Stereoanomaly. Richards (1970) reported deficient stereopsis in some $30 \%$ of the population, with greater impairment in the processing domain responsible for uncrossed RDs (and, therefore, crossed FDs; see also Richards, 1971a). This asymmetry may reflect a general advantage for crossed RDs in stereofusion.

Developmental priority. The processing of both crossed and uncrossed RDs is achieved in the first year of life, but crossed RDs appear to have developmental priority (Birch, Gwiazda, \& Held, 1982; Held, Birch, \& Gwiazda, 1980).

Sex differences. A sex difference exists in the perception of random-dot stereograms: Both males and females tend to be more sensitive to crossed RDs, with this pattern being more marked in females - that is, they have a lower threshold for perceiving depth in a random-dot stereogram in the crossed case (Zaroff, Knutelska, \& Frumkes, 2003).

We have seen that there is a clear asymmetry between the processing associated with crossed and uncrossed RDs, with the former facilitating fusion more than the latter. This conclusion suggests that crossed RDs (equivalent to uncrossed FDs) should be preferred when stimulus conditions are such as to jeopardize binocular fusion; as shown below, uncrossed FDs will also perforce occur in very close viewing at the limits of convergence. We assume that binocular fusion difficulty is at least partly defined as resulting from stimulus conditions that militate against solving the correspondence problem (i.e., coordinating the images from the two eyes): These visual stimulus conditions will include low contrast, absence of large distinct areas and contours, and a low figure-to-ground ratio. We have seen that uncrossed FDs in reading studies are more associated with bright text on a dark background, subtending a small retinal angle in dark conditions (Figure 1). However, it does not necessarily follow that these uncrossed FDs are a consequence of fusion difficulty. Is 
there any experimental evidence that viewing conditions militating against binocular fusion can precipitate crossed RDs? Several studies support this conclusion.

Jaschinski-Kruza (1994) reported that reduced luminance and increased target blur cause a shift to uncrossed FDs, where the fixation target was a block of text (see, also, Pickwell, Jenkins, \& Yekta, 1987); this change in disparity was up to 20 arc min in participants whose angle of resting vergence in dark conditions (i.e., dark vergence) was smaller (i.e., more distant) than was the viewing distance used (Jaschinski, 1997). Richards (1973) reported the case of several stereo-anomalous observers, for whom the sign of their perceived depth confusion (mistaking zero-disparity for crossed or uncrossed RDs) was reversed in disparity when the stimuli changed from dark bars on a light background to light bars on a dark background. We are currently exploring a single case of a normal reader's changes from crossed to uncrossed FDs in reading when room illumination changes from normal to low level, with black text on a white screen (Shillcock, Roberts, Kreiner, \& Mac Cumhaill, 2007). ${ }^{11}$ Thus, uncrossed FDs (crossed RDs) are not just associated with more robust fusion; there are grounds for believing that the relevant conditions can precipitate uncrossed FDs.

In overall summary, we have seen a general preference for crossed RDs over uncrossed RDs (uncrossed FDs over crossed FDs) in nonreading tasks, where fusion is more or less directly implicated. We might, therefore, expect uncrossed FDs to predominate in reading. However, the studies listed in Table 1 do not unambiguously confirm this prediction. In fact, all the studies reported some percentage of crossed FDs and, in some cases, this percentage is greater than for uncrossed FDs. Next, we explore the roles of uncrossed RDs or crossed FDs in nonreading tasks in the light of the previous review, before considering why FDs in reading are not universally uncrossed.

\section{Advantages of Uncrossed RDs in Nonreading Tasks}

We have shown that crossed RDs (uncrossed FDs) are advantageous in several different ways. However these advantages are not exclusive, as shown below: Uncrossed RDs (crossed FDs) have at least some advantages over crossed RDs (uncrossed FDs), although these advantages may be relatively minor in the case of reading.

Asthenopia. Near-vision tasks tend to generate uncrossed FDs when the convergence response is weak and vergence tends toward the (distant) resting position; as a result, crossed FDs and aligned binocular fixations as a group are associated with less asthenopia (visual stress caused by near-vision tasks) than are uncrossed FDs (Jenkins, Pickwell, \& Yekta, 1989; Pickwell, 1989, 1991; see, also, Jainta \& Jaschinski, 2002; Jaschinski, 1997, 2001), although there are individual differences (Pickwell, 1989). Further, eye strain in close work is stronger in those subjects with marked changes in FD in the uncrossed direction due to near vision (Jaschinski, 2002). Uncrossed FDs appear to be more muscularly onerous for the visual system than are crossed FDs (although there may be an issue of cause and effect if uncrossed FDs are a response to other problems for binocular fusion).

Temporal aspects of $R D$ processing. There is an initial bias toward uncrossed RDs. Goutcher and Mamassian (2006) recorded crossed or uncrossed RD perceptual reports over time, as participants stared at a stereoscopically bistable stimulus. They found an initial preference for uncrossed RDs, followed by alternating perceptions, and concluded that there seem to be two distinct phases in stereo matching - one early and the other sustained.

An initial preference for crossed FDs was also reported by Setter and Norman (2006) in a series of nonreading experiments in which random-dot stereograms were presented at RDs that corresponded to being in front of, on, or behind the screen. The authors reported that their 7 participants first fixated the screen briefly (never less than $50 \mathrm{msec}$, the minimal time required to perceive a random-dot stereogram; Julesz, 1964; Tyler, 1991) and then converged, so that the principal lines of sight crossed in front of the screen. Furthermore, this point of (crossed) convergence was closer to the observer for crossed retinal images, was farthest away from the observer for uncrossed retinal images, and was at an intermediate distance when the image disparity was zero. The authors discussed their results in terms of the system reverting to the resting point of vergence (cf. Jainta \& Jaschinski, 2002; Jaschinski, Bröde, \& Griefahn, 1999; Jaschinski, Koitcheva, \& Heuer, 1998; Jaschinski-Kruza, 1994).

Viewing distance. The disparity limit for crossed RDs is unaffected by angular size, but, for uncrossed RDs, it tends to increase with increasing convergence (decreasing fixation distance; Richards, 1971b). Furthermore, Richards and Foley (1971) found that stereoacuity increased with accommodation for uncrossed RDs, but not for crossed RDs. Uncrossed RDs, thus, seem more flexible for near viewing.

A review of the literature shows that asthenopia, temporal aspects of $\mathrm{RD}$ processing, and viewing distance exhaust the case that can be made for specific relative advantages of uncrossed RDs in nonreading tasks. Only asthenopia and viewing distance seem to speak to the task of reading, although not strongly: None of the reported analyses of FD in reading suggest that fatigue plays an important role, and Figure 1 shows that uncrossed FDs pattern with the smaller stimuli that might be expected to make more demands on stereoacuity. How can we account for those data sets that show very substantial proportions of crossed FDs? We now explore the ways in which the perceptual apparatus between the cornea and the cortex-the fovea and the visual pathways - offer real postfusional ${ }^{12}$ processing advantages to crossed FDs in reading and play a major role in the theory of foveation that we advance.

\section{A Model of Binocular Foveation}

We have seen that uncrossed FDs can be expected to facilitate binocular fusion in reading, but that they are far from universal in reading (Figure 1). We hypothesize a complementary, postfusional (or nonfusional) processing advantage from crossed FDs in reading conditions that do not jeopardize binocular fusion; that advantage is based 
on the prioritized processing afforded to the temporal hemifield and nasal hemifovea (e.g., Toosy et al., 2001), which we explore below. We present a model that combines four influences on the quality of the visual input that is presented to postfusional processing (i.e., to word recognition and other aspects of reading): (a) precision of foveal splitting, (b) contralateral preference in the visual pathways, (c) ocular prevalence, and (d) FD. Figure 4 shows details of the projection of the word intermediate to the vertically divided human fovea of each eye, with an FD of 2 letters. Crossed and uncrossed versions of the FD are shown in (a) and (b), respectively. Each foveal window receives information from 12 letters -6 to the right and 6 to the left of fixation. Bold type denotes the advantageous contralateral projection from a particular eye to the op- posite hemisphere, and regular type denotes an ipsilateral projection. Each eye projects to both hemispheres: The RVF projects to the left hemisphere (LH), and the left visual field (LVF) projects to the right hemisphere (RH). We now introduce the four factors listed above.

Precision of foveal splitting. Our theory of foveation proceeds from the fact that the human fovea is vertically divided, projecting to the cerebral hemispheres in the same way as does the extrafoveal retina (Brysbaert, 1994, 2004; Ellis, 2004; Ellis \& Brysbaert, 2010; Lavidor \& Walsh, 2004; Leff, 2004; McDonald \& Shillcock, 2005a, 2005b; Monaghan \& Shillcock, 2008; Monaghan, Shillcock, \& McDonald, 2004; Shillcock, Ellison, \& Monaghan, 2000; but see Jordan \& Paterson, 2009). In previous modeling research, we have assumed a simple, precise, vertical split

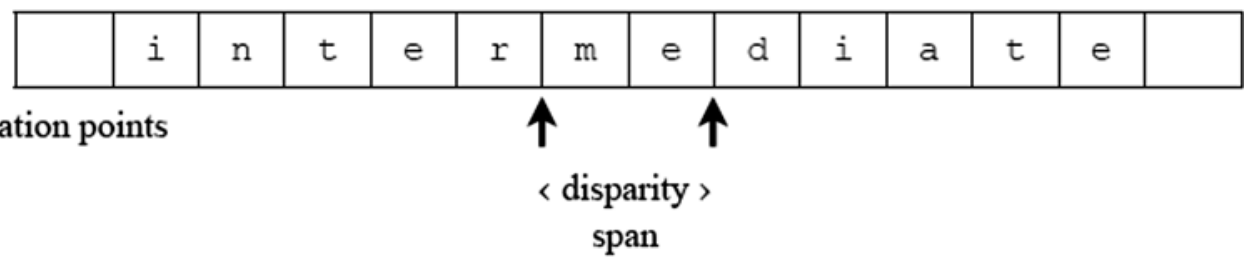

\section{A. Crossed fixations:}

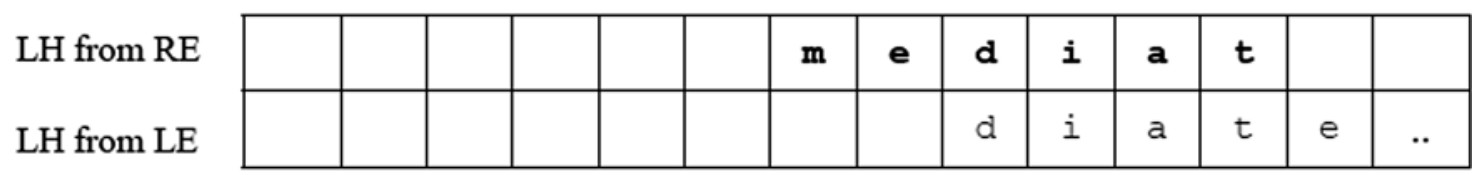

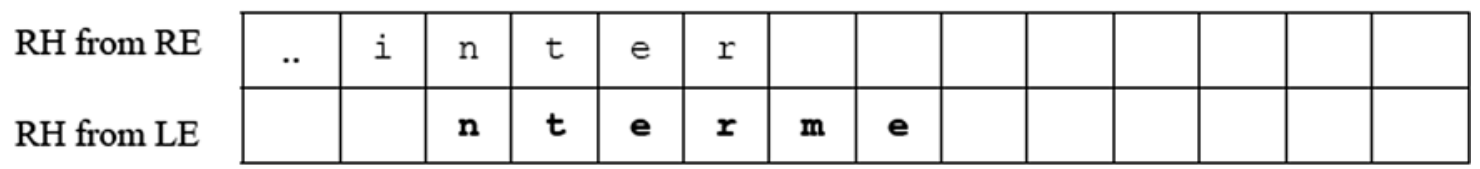

\section{B. Uncrossed fixations:}

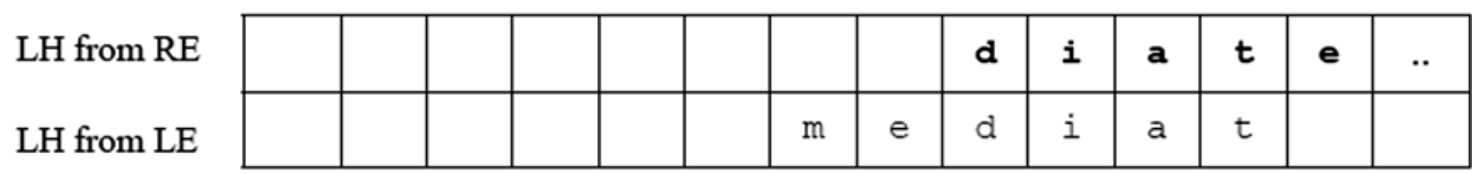

RH from RE

RH from LE

\begin{tabular}{|l|l|l|l|l|l|l|l|l|l|l|l|l|l|}
\hline & & $\mathrm{n}$ & $\mathrm{t}$ & $\mathrm{e}$ & $\mathrm{r}$ & $\mathrm{m}$ & $\mathrm{e}$ & & & & & & \\
\hline $\boldsymbol{*}$ & $\mathbf{i}$ & $\mathbf{n}$ & $\boldsymbol{t}$ & $\mathbf{e}$ & $\boldsymbol{r}$ & & & & & & & & \\
\hline
\end{tabular}

Figure 4. The contralateral preference and the consequences for processing and representation in crossed and uncrossed FDs, with a foveal window of six characters to either side of each eye's fixation point and a simplifying assumption of uniform acuity within the foveal window. The arrows denote the fixation points of the two eyes. There is an FD of two characters. Bold type denotes prioritized processing due to contralateral projection; ordinary type denotes ipsilateral projection. See the text for details. 
at the foveal midline (see, e.g., Shillcock et al., 2000) in order to explore the implications of the abstract division of input between the two halves of a divided processor. However, the functional anatomy of the human fovea is the focus of continuing research. The issue is the precision of the divided anatomical projection from the fovea, and this question is not straightforwardly settled solely by anatomical investigation, because of the limits on precision of the relevant techniques. Reinhard and TrauzettelKlosinski's (2003) data suggest individual variation in the precision of foveal splitting, with a majority (two-thirds) between $0^{\circ}$ (precise splitting) and $0.3^{\circ}$, and a minority case of an overlap tending toward $0.6^{\circ}$ (i.e., a total overlapping section of $1.2^{\circ}$ ). In Figure 4, we have shown simple, precise, foveal splitting. Below, we will model this range of precision in foveal splitting.

The contralateral preference. Contralateral projection is a pervasive principle in the human visual system; the RVF initially projects to the LH, and the LVF initially projects to the RH, in each eye. Toosy et al. (2001) reported finding a neurophysiological bias between these two pathways. They show that monocular Ganzfeld stimulation of one eye causes greater cortical activation in the contralateral hemisphere than in the ipsilateral; the left eye causes more activation in the $\mathrm{RH}$, and the right eye causes more activation in the LH, even though both eyes project to both hemispheres. This observation may reflect a bias at several different levels, as discussed by Toosy et al.: unique contralateral representation of a crescent at the temporal hemifield (Horton \& Hocking, 1996), greater retinal ganglion cell density in the nasal hemiretina (corresponding to the temporal visual hemifield; Perry \& Cowey, 1985), a bias in favor of crossed fibers at the optic chiasm (Kupfer, Chumbley, \& Downer, 1967) and at the lateral geniculate nucleus (Chacko, 1948), organizational bias in the ocular dominance columns in V1 in favor of contralateral input (LeVay, Connolly, Houde, \& Van Essen, 1985), and greater contralateral contribution to the activity of V1 cells with a binocular receptive field (Toosy et al., 2001). In short, the human visual system prioritizes processing of the contralateral input at multiple stages, from cornea to cortex. We are currently exploring whether this contralateral preference also applies to foveal processing of orthographic stimuli, using a haploscope to control presentation of the two halves of a word to separate hemispheres (see Obregón \& Shillcock, 2007, 2010). ${ }^{13}$

Ocular prevalence. The phenomenological prioritization of the signal from one eye over that from the other eye manifests itself as ocular dominance when fusion is impossible or problematic (as in ocular rivalry paradigms) and as ocular prevalence when fusion is assured (Kommerell, Schmitt, Kromeier, \& Bach, 2003). Ocular dominance and ocular prevalence are both operationally defined within particular studies, typically using sighting tests and stereoscopic alignment, respectively. In ocular dominance, the right eye is more often preferred over the left ( $40 \%$ compared with $20 \%$; Hillemanns, 1927). In ocular prevalence, which is more relevant to reading (in which fusion is typically assured), the right and left eyes are equally likely to be preferred in different individuals (Kommerell et al., 2003). Most people exhibit some level of ocular prevalence (Ehrenstein, Arnold-Schulz-Gahmen, \& Jaschinski, 2005; Erkelens, Muijs, \& van Ee, 1996; Haase, 1995; Heinrich, Kromeier, Bach, \& Kommerell, 2005; Jaschinski \& Schroth, 2008; Kommerell et al., 2003; Sachsenweger, 1958). What we wish to capture by the term ocular prevalence is the "graded quantification of the balance between the eyes" (Kommerell et al., 2003, p. 1397) that is achieved in conditions of fusion with no diplopia; such a quantitative, graded difference in cortical activation has been reported for dominance (Rombouts, Barkhof, Sprenger, Valk, \& Scheltens, 1996), at least partly grounded in anatomy and physiology. In the model of foveation in reading reported below, ocular prevalence means that one eye will have more effect than the other on cortical representation, even though (unlike the standard operational definition of ocular prevalence) the input from the two eyes is from the same stimulus at the same depth.

FD. In the idealized projections, shown in Figure 4, we have assumed a precise foveal split at the fixation point of each eye to emphasize the different patterns of contralateral and ipsilateral projections between the crossed and uncrossed FDs in Figures 4A and 4B. The crossed FD combines two nasal hemifoveal projections at its center (i.e., between the two fixation points, the disparity span), via the preferred contralateral channels. The uncrossed FD combines two temporal hemifoveal projections at its center via the dispreferred ipsilateral pathways. The mean size of the disparity span for disparities greater than one character varies between studies, from a minimum of just over one character in Liversedge et al. (2006). Such a figure is the mean of a distribution over all reported participants; the distribution in the general population over all reading conditions will contain larger disparities, necessarily from the large-disparity tail of the distribution and possibly from reading conditions that elicit larger disparities.

Modeling the visual pathways. We have motivated the inclusion of four variables in the model: precision of foveal splitting, contralateral preference, ocular prevalence, and FD. Our model of the visual pathways allows us to explore the interaction between these variables in terms of the input to the word-recognition processes. In the model, depicted in Figure 5, each eye is represented by an idealized, one-dimensional retina, in which acuity diminishes linearly with eccentricity (Anstis, 1974; Beard, Levi, \& Klein, 1997; Jacobs, 1979; Levi, Klein, \& Aitsebaomo, 1985; O’Regan, Lévy-Schoen, \& Jacobs, 1983). Text is projected onto this gradient. Contralateral and ipsilateral projections are determined with respect to each retinal midline, and the extent of the bilateral projection resulting from imprecise foveal splitting. These projections are further modulated by the degree of ocular prevalence and by contralateral preference. Representations of the projected text are computed for the left and right hemispheres by fusing the two eyes' projections of the LVF/RH and RVF/ LH, respectively. Finally, the two hemispheric representations are fused to form a coordinated interhemispheric representation of the text. We have coined the term repre- 


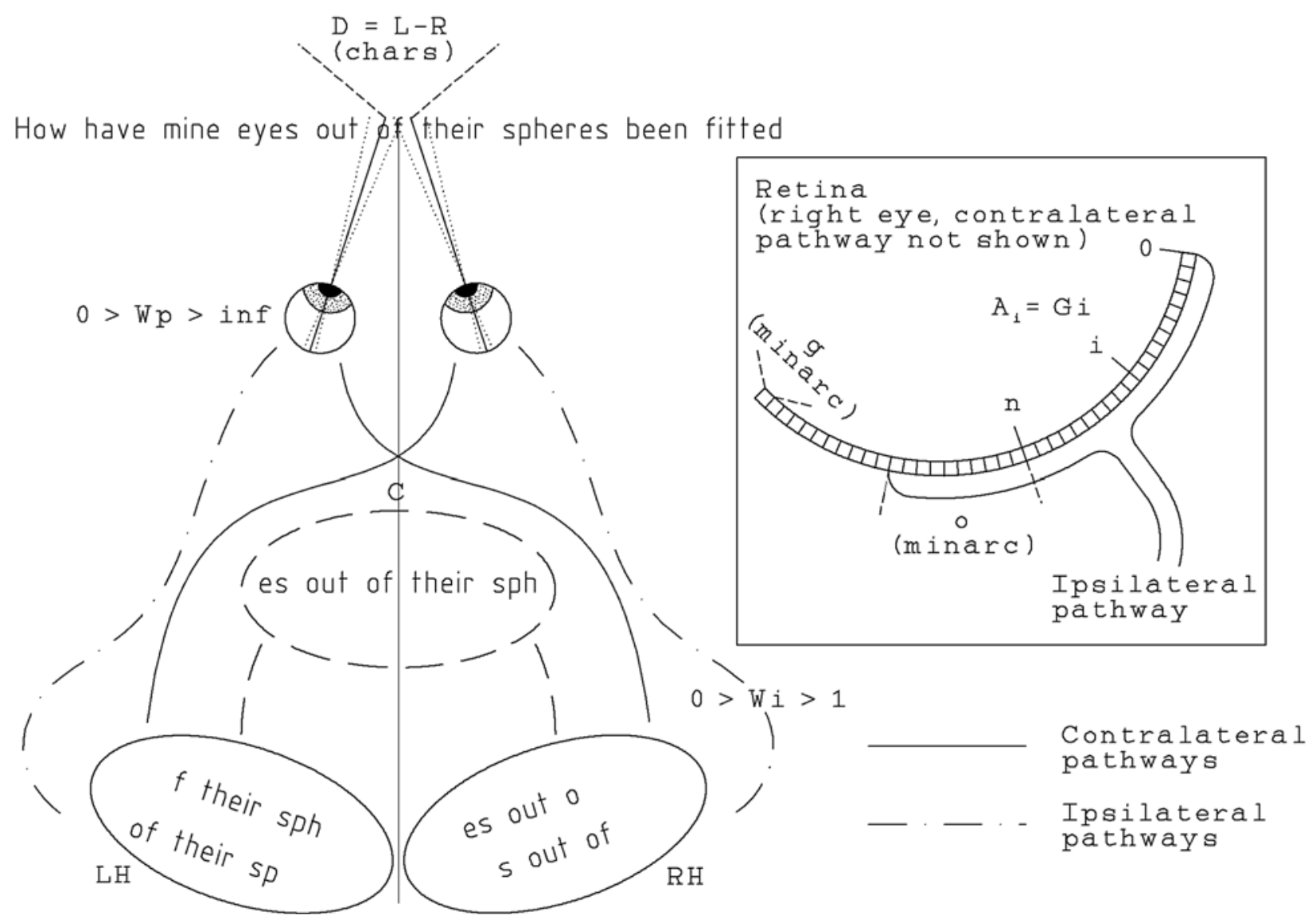

Figure 5. A model of activation in the visual pathways, elaborating the model in Figure 4. The retina is modeled as a one-dimensional vector of length $n$, with each value representing mean acuity within $g$ arc min of distance. Acuity $A$ diminishes linearly from the center with gradient $G$. Projection of text onto the fovea is modeled as activation equivalent to the acuity in each division, with activation set to 0 where no text is projected. Ocular prevalence is modeled as a weighting on input through the left eye $\left(W_{\mathrm{p}}\right)$; values of less than 1 represent right-eye prevalence. Activation is propagated to the cortex via contralateral (e.g., right eye to left hemisphere [LH]) and ipsilateral (e.g., right eye to right hemisphere [RH]) pathways. Temporal activation is transferred ipsilaterally, and nasal activation is transferred contralaterally, with each pathway overlapping the retinal midline by $o$ arc min. Contralateral preference is modeled by a weighting on the input through the ipsilateral pathways $\left(W_{\mathrm{i}}\right)$. Binocular fusion is modeled as occurring in the $\mathrm{LH}$ and $\mathrm{RH}$ by aligning and summing the separate inputs from the two eyes; fused representations are coordinated in a similar manner $(C)$. Variation in FD $(D), W_{\mathrm{p}}, W_{\mathrm{i}}$, and $o$ results in different patterns of activation in the coordinated representation.

sentational activation to describe the numerical distinctions and differences embodied by our model of the visual pathways. This term is intended to be theoretically neutral and can be interpreted in several ways. Since the model begins with the acuity gradient at the retina, one interpretation is that differences in representational activation are essentially differences in acuity, with greater activation being equivalent to greater representational detail. An alternative possibility is that representational activation is simply greater cortical activation, following the finding of Toosy et al. (2001) that greater cortical activation is found in the contralateral hemisphere. Irrespective of precisely what representational activation represents, it is intended that a greater quantity of it provides more useful input to postfusional cognitive processes. In reading, greater representational activation should, therefore, facilitate postfusional processing, ceteris paribus.

Our observations concerning the four variables lead to the hypothesis that crossed FDs produce higher rep- resentational activation near the points of fixation than uncrossed FDs (cf. Figure 4). Simulations with our model (Figure 5) confirm that this is robustly the case.

\section{Simulations}

Figures 6-9 illustrate the behavior of the model in four sets of simulations. In each graph, the $x$-axis represents the horizontal extent of the word "intermediate," with representational activation on the $y$-axis. Each simulation explores one of the four variables discussed above, and all of the simulations contrast the three types of FD: crossed, uncrossed and conjoint. Nonconjoint FDs are of two characters, except in Figure 9, in which a larger range of FDs is explored. Arbitrary default values of variables shown in Figure 5 were $g$ (grain) $=1$ arc min and $G$ (acuity gradient $)=1$. In addition, the length of the vector of values being considered: $n=150$ (i.e., $2.5^{\circ} \times 60$ arc $\mathrm{min}$, the approximate extent of the fovea); $o$ (degree of bilateral overlap) $=10 \operatorname{arc} \min$ (i.e., $0.15^{\circ}$, the median value of the ma- 


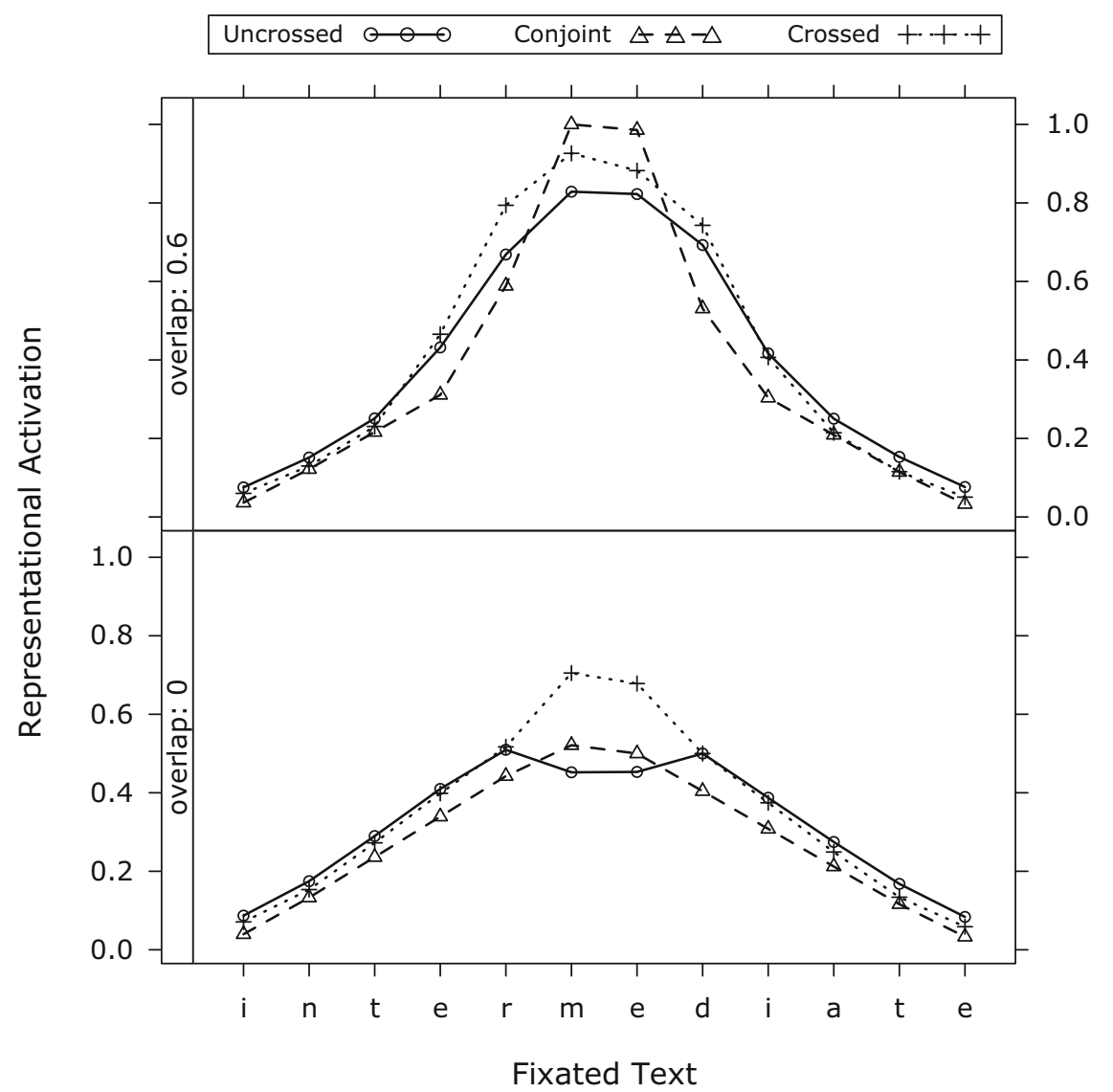

\begin{abstract}
Figure 6. Qualitative differences among crossed, conjoint, and uncrossed FDs, for zero and $0.6^{\circ}$ overlap (i.e., bilateral projection) within each fovea. In this graph and later ones, "representational activation" stands for an unspecified dimension of acuity and/or cortical activation, which facilitates lexical access. $D=\{-2,0,2\}$ characters; $W_{\mathrm{p}}=.9 ; W_{\mathrm{i}}=.7$. Minor asymmetries in activation result from ocular prevalence. See the text for details of parameters.
\end{abstract}

jority case reported by Reinhard \& Trauzettel-Klosinski, $2003) ; W_{\mathrm{p}}$ (ocular prevalence $)=.9$ (weak right-eye prevalence $) ; W_{\mathrm{i}}($ contralateral preference $)=.7 ; D$ (disparity $)=$ $\{-2,0,2\}$ (relatively large, to emphasize differences between crossed, conjoint, and uncrossed fixations). Each character subtended $0.43^{\circ}$. Resolution (of foveal bins to characters), binocular fusion, and coordination were all done by taking mean values. Representational activation is normalized in the range $0-1$. See the caption of Figure 5 for more details.

Precision of foveal splitting. Figure 6 shows the results of modeling the smallest overlap at $0^{\circ}$ (precise splitting, bottom panel) and the largest overlap at $0.6^{\circ}$ (i.e., a total bilateral projection of $1.2^{\circ}$; cf. Reinhard \& Trauzettel-Klosinski, 2003). When foveal overlap is 0 (precise splitting), the crossed FDs produce better results than do uncrossed FDs within the disparity span, with conjoint fixations producing intermediate results. When bilateral projection is increased, the difference in representational activation between crossed and uncrossed FDs is reduced but spreads outside the disparity span, with crossed FDs still higher. Within the disparity span, activation for conjoint fixations increases to a greater ex- tent than does activation for either crossed or uncrossed fixations.

Contralateral preference. The contralateral preference was operationalized as a weight on the ipsilateral pathways in the range $0<W_{\mathrm{i}}<1$. Figure 7 compares the effects of three levels of contralateral preference. Variation in the degree of contralateral preference has opposing effects in crossed and uncrossed FDs. A strong contralateral preference ( $W_{\mathrm{i}}=.2$, lowest panel) increases representational activation near the points of fixation in a crossed FD, but depresses it in an uncrossed FD. Although less marked, this difference is still apparent, with a relatively weak contralateral preference $\left(W_{\mathrm{i}}=.8\right.$, top panel).

Ocular prevalence. Figure 8 shows a comparison of the effects of three levels of ocular prevalence. Ocular prevalence was operationalized as a weight on the left eye $\left(W_{\mathrm{p}}>0\right)$. Strong ocular prevalence tends toward a monocular fixation, the input being dominated by one eye. Thus, the greatest degree of ocular prevalence $\left(W_{\mathrm{p}}=.1\right.$, lowest panel) diminishes the difference in representational activation between the three fixation types in the disparity span. In addition, the peak of representational activation corresponds to the right eye in the three types of FD (the 


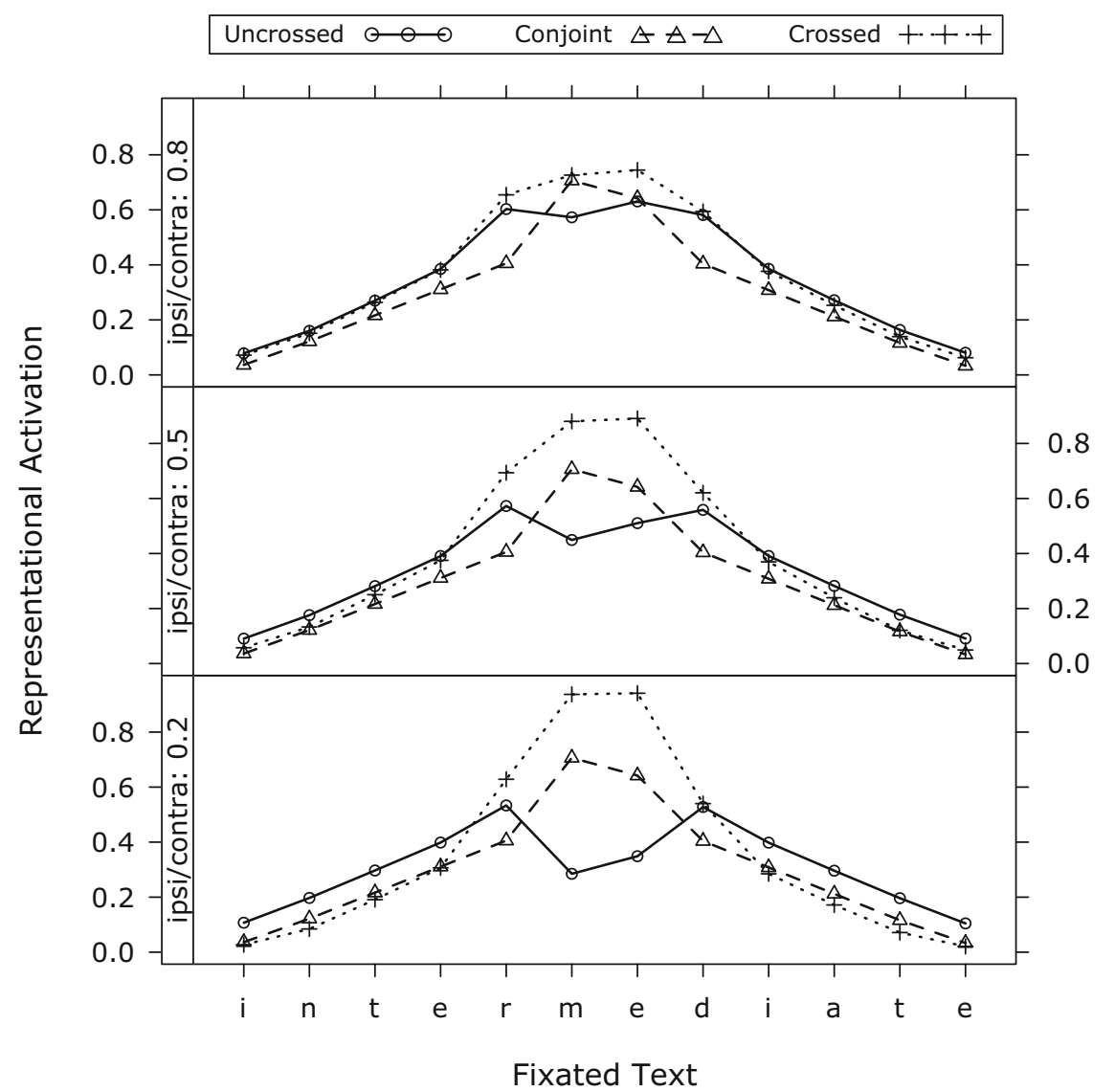

Figure 7. Qualitative differences among crossed, conjoint, and uncrossed FDs, for differing contralateral preference ratios. $D=\{-2,0,2\}$ characters; $W_{\mathrm{p}}=.9 ; o=10$ arc min.

opposite pattern would be observed for left-prevalent individuals). Smaller degrees of ocular prevalence ( $W_{\mathrm{p}}=.5$, center panel; $W_{\mathrm{p}}=.9$, top panel) introduce further differences and asymmetries between crossed and uncrossed FDs in the disparity span, as the (weak) contralateral preference exerts a more noticeable effect.

FD. FD was specified in characters. Figure 9 depicts the variation in representational activation resulting from nine levels of FD. Clear differences between crossed and uncrossed FDs emerge. First, the general level of activation around the eyes' fixation points is substantially greater for crossed FDs. Second, as FD increases within each of the crossed and uncrossed panels, activation begins to fall in the center of the disparity span (as defined in Figure 4). This diminution is to be expected, but these simulations show that it is deeper and appears at smaller disparities in the uncrossed FDs.

A final important aspect of the modeling shown in Figure 9 is that both types of FD (top and bottom panels) produce wider plateaus of higher levels of representational activation than in the conjoint case (albeit there is a dip in the middle of the larger uncrossed FDs). This effect constitutes a general advantage for FDs of both types: FD extends the width of the foveal window by offsetting the foveal windows of the two eyes, so that more of the text falls within high-acuity vision. The direction of the
FD determines whether it is the privileged nasal or the nonprivileged temporal hemiretinas/hemifoveas that are overlapped. The implications for reading will be mediated by specific and lexical attentional factors (cf. Henderson \& Ferreira, 1990), but one testable prediction is that saccade length should be extended for larger disparities (see Roberts et al., 2010). ${ }^{14}$

In summary, robust processing differences emerge in simulating crossed and uncrossed FDs, reflecting the roles of degree of foveal splitting, of contralateral preference in the visual pathways, of ocular prevalence, and of FD. We have demonstrated that clear and pervasive advantages result from crossed FDs. In all the simulations, only conjoint fixations ever emerged as more advantageous than crossed FDs, and then only in a small minority of simulations. It is worth noting that explicit computational modeling was required to make these complex binocular interactions interpretable; these predicted differences between crossed and uncrossed FDs could not have arisen from ad hoc theorizing.

\section{Binocular Disparity in Reading}

An uncrossed FD impairs fusion less than the samesized crossed FD does, and conditions in which fusion is difficult produce a greater degree of uncrossed than of crossed FDs. In addition, a combination of established 


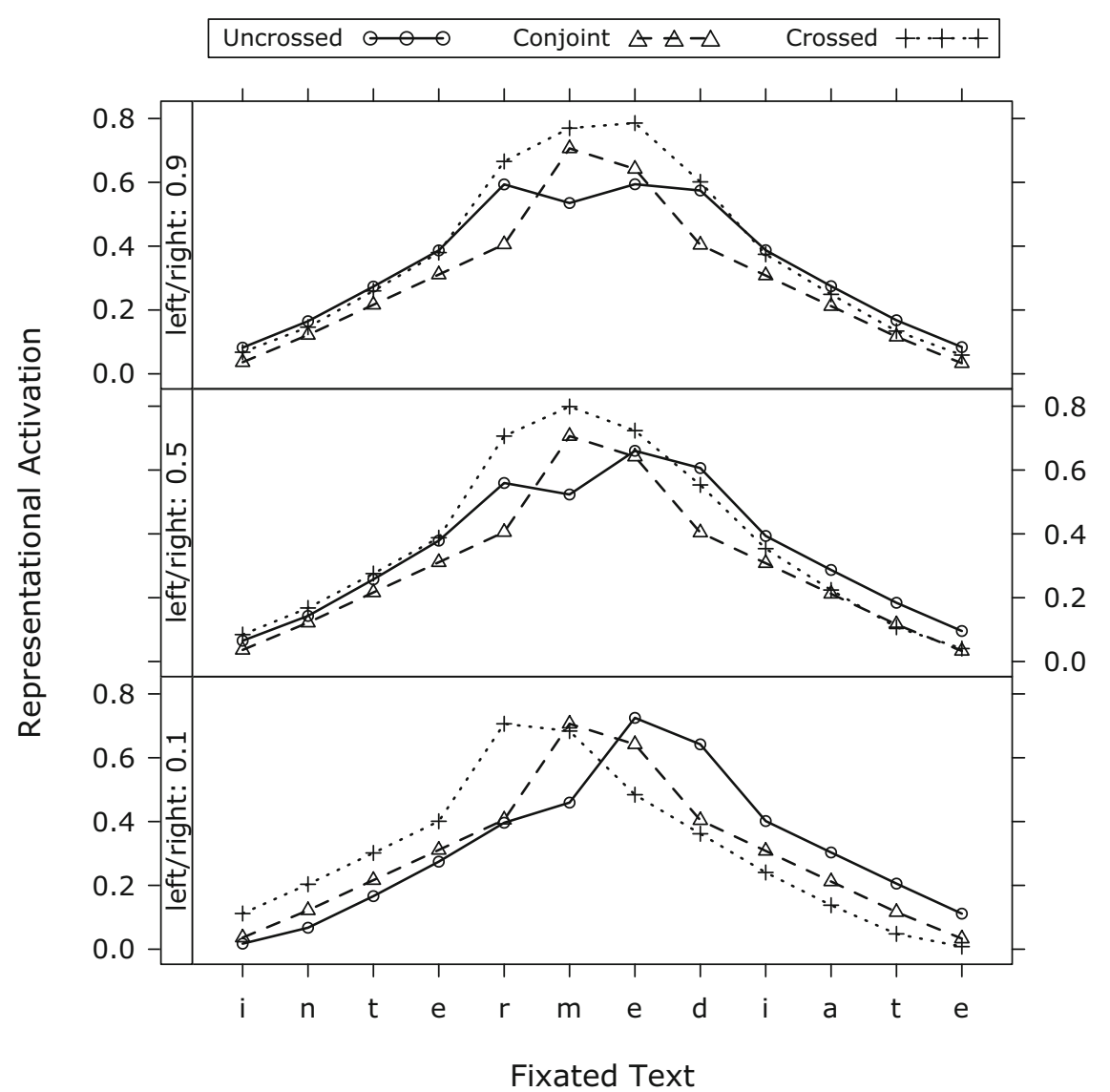

Figure 8. Qualitative differences among crossed, conjoint, and uncrossed FDs, for different ocular prevalence ratios. $D=\{-2,0,2\}$ characters; $W_{\mathrm{i}}=.7 ; o=10$ arc min.

findings about the visual pathways suggest that crossed FDs provide a processing advantage in the form of greater representational activation around, and especially between, the points of fixation, as our model illustrates. Our principal claim is that these two observations together explain the variation in FD between the studies of binocular reading shown in Figures 1 and 2. Figure 10 illustrates this idea. Temporal vergence (divergence, uncrossing in reading) is excited by fusion-related activity: When significant effort is expended to ensure binocular fusion or when fusion is uncertain, the saccade generator produces temporal vergence. Nasal vergence (convergence, crossing in reading) is excited by postfusion activity: The more work the processor is required to do, the more it "requests" crossing to alleviate this load.

This model makes several strong predictions about future experimental data. First, it predicts that, when fusion conditions are difficult in reading, more uncrossed FDs will be observed. Further research is required to delineate the conditions that cause fusion difficulty in reading, but work on nonreading tasks, using paragraphs of text as stimuli, has shown that background luminance and text blur increase the tendency toward uncrossed FDs. As noted, we are currently exploring a single case study of the effects of differing illumination on reading (Shillcock et al., 2007). In addition, the effect on FD of the angle subtended by text at the retina is the subject of ongoing investigation (Roberts, Kreiner, Obregón, \& Shillcock, 2009; Yen \& Radach, 2007); the meta-study reported in Figure 1 apparently shows such an effect. These variables would, therefore, seem to be a good starting point for investigation. Second, the theory predicts that, when fusion is unproblematic, crossed FDs will facilitate lexical processing to a greater degree than will uncrossed FDs. Although straightforward, this prediction will, we suggest, require very careful falsification, given that an extended fixation duration may potentially be caused by problematic fusion processing and/or by problematic postfusion processing, with both of these sources of variation subject to individual differences.

\section{Discussion}

We have tried to address two research communities: the relatively small one concerned with binocular reading and the large vision science community concerned with binocularity in general and with vergence and depth perception in particular. To clarify our position, we summarize it as follows:

1. The pervasive departures from exactly conjoint binocular fixations in reading should not simply be dismissed as error or noise; rather, such absolute disparities, or “overall disparities” (Howard \& Rogers, 1995, p. 243), 


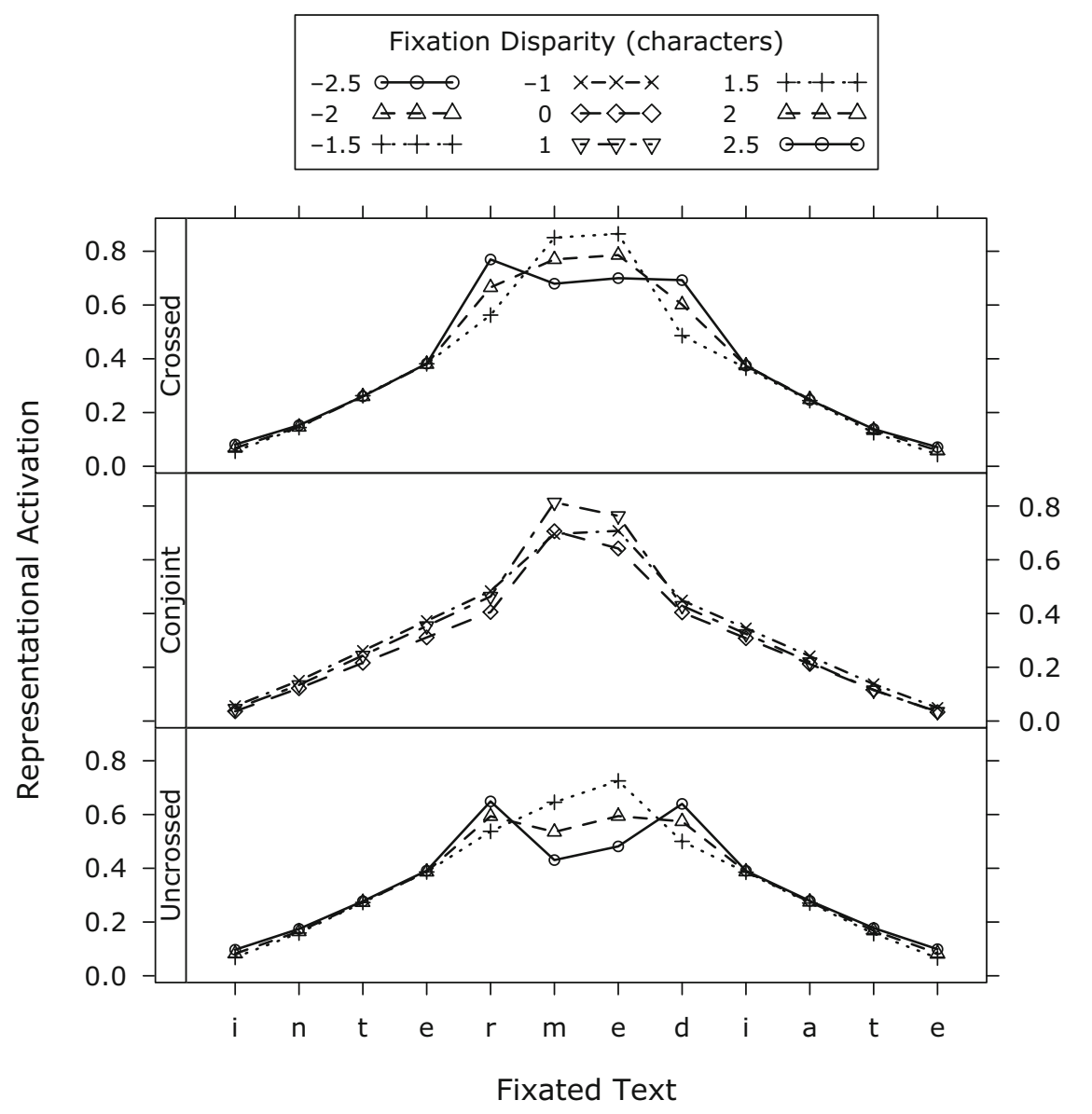

Figure 9. Qualitative differences among crossed, conjoint, and uncrossed FDs, for different binocular disparities. Positive numbers refer to crossed $\mathrm{FDs}\left(W_{\mathrm{p}}=.9, W_{\mathrm{i}}=.7, o=\right.$ 10 arc min).

in reading can be interpreted as adaptive, regardless of their origin.

2. Such interpretations are required for both directions of horizontal departure (i.e., right eye's fixation point to the left or right of the left eye's fixation point on the text) from exactly conjoint binocular fixation. These interpretations need to be congruent with the substantial existing data for nonreading as well as reading tasks.

3. Placing the intersection of the principal visual axes, or lines of sight, in front of the text or (virtually) behind the text, respectively, has the effect of placing the text behind or in front of the horopter.

4. These two regions (behind or in front of the horopter) are associated with different behaviors in depth perception, with the coordination of retinal images from objects away from the horopter; we update Mustillo's (1985) little-cited review of processing in the two depth-perception regions.

5. Although absolute disparity (or overall disparity, the angle between the two principal visual axes) is not understood to be used on its own to gauge depth accurately for a single point, the core behavior of the eyes is their ability to converge on an isolated point, meaning that information about absolute disparity is available. Indeed, V1 binocular neurons specialize in absolute, not relative, disparity.
6. The general absence of depth in reading (i.e., relative disparity between points at different depths) does not mean that the resources and mechanisms associated with binocular depth processing are not available and are not engaged. The computational problem of depth perception is one of coordination of the images on the two retinas.

7. Both eyes contribute in binocular reading; monocular reading is less effective.

8. Reading requires the coordination of visual information on the two retinas. Our review of the depth perception literature confirms that there is robustly better coordination in depth perception when relative disparity involves one direction of departure from conjointness - that is, for the images of an object between the viewer and the horopter (crossed RD). This advantage is equivalent to a processing advantage for noncorresponding images in reading in which the left eye's fixation point is to the left of the right eye's fixation point (uncrossed FD).

9. A different processing advantage for images in the other direction of departure from exactly conjoint binocular fixation (left eye's fixation point to the right of the right eye's fixation point) is suggested by computational modeling that we report, involving a range of physiological and anatomical influences between the cornea and the cortex. 


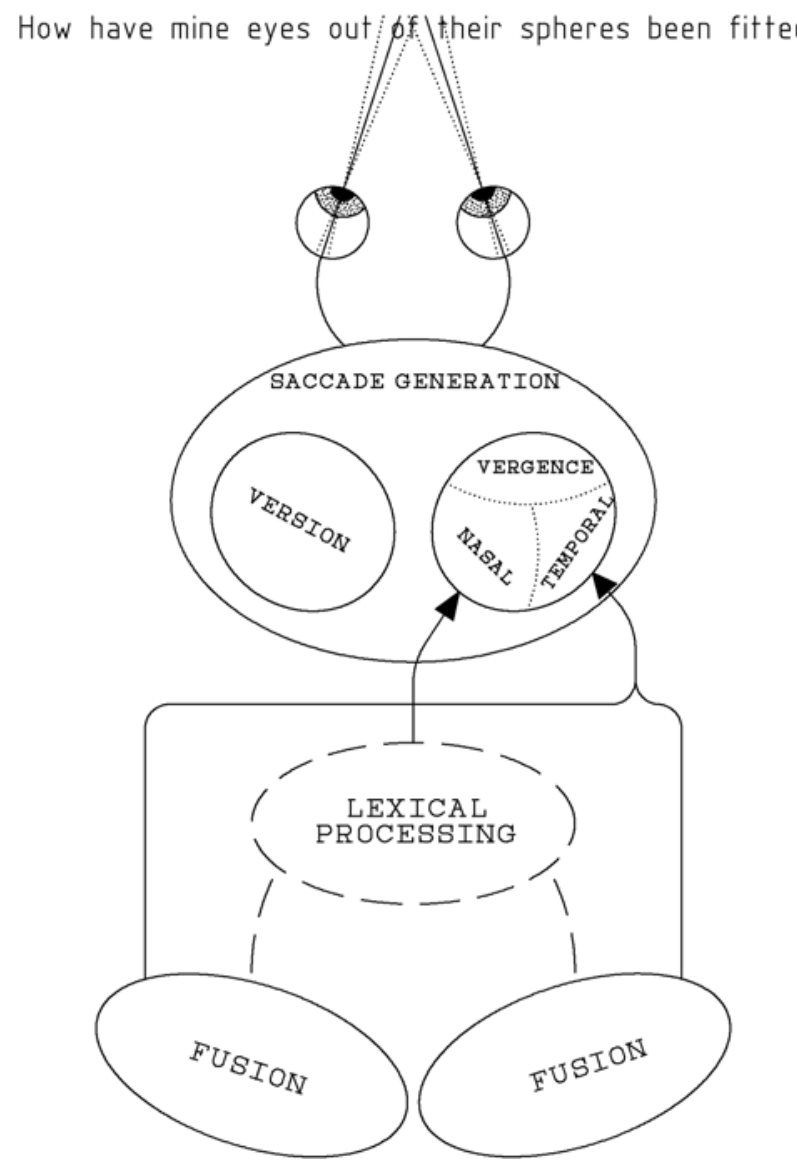

Figure 10. Schematic model of binocular fixation in reading. Temporal vergence (divergence) in saccade generation is excited by hemispheric fusion processes; problematic fusion may be offset by creating uncrossed FDs. Nasal vergence (convergence) is excited by activity in postfusion processing; higher cognitive processing, such as the requirement to expand the perceptual span to accommodate more words, may precipitate crossed FDs.

10. We relate the two different advantages described in Points 8 and 9 with stimulus conditions reported in the literature. This is formalized in a model of how the two eyes foveate text in reading.

We have argued for a particular interpretation of binocular foveation behaviors on the basis of the existing literature on depth perception and on the processing implications of the visual pathways. We have formulated these interpretations in terms of the combination of the influences of binocular FD, ocular prevalence, contralateral preference, and degree of foveal bilateral projection (Figure 5) and in the hypothesized relationships between divergence and fusional processing difficulty and between convergence and postfusional processing difficulty (Figure 10).

A methodological assumption in this theorizing has been that unexpected behavioral data (such as the very existence of binocular FD) may be in some way adaptive, rather than being solely the result of "noisy" processing in the reader, of artifact in the equipment, or of experimenter error. We have shown ways in which binocular FD can be adaptive, both in its size and direction (crossed or uncrossed).
Our theory of binocular foveation has shown that it is possible to see binocular vision not primarily in terms of the problems of alignment and correspondence but rather, in terms of a remarkable flexibility in responding to variation in the visual input. An important principle here is that the visual system achieves this flexibility by a peripheral mechanism of vergence: Converging or diverging the eyes tips the visual system into different modes of operation, taking advantage of different processing domains within the visual system. A problem of optimizing the processing of the visual input is solved in a mechanical, embodied manner, as opposed to one that is purely abstract and computational.

The theory of binocular foveation we have presented makes a number of empirical predictions that require careful testing. These predictions are probably better assessed by large corpora of naturalistic eye movements than by factorial experiments. Two principal issues militate against reliance on factorial experimentation and in favor of the greater statistical power available from large corpus analysis: the impossibility of manipulating disparity directly as an independent variable in text reading and the complex confounds introduced by the interconnection and intercorrelation of the various factors that affect binocular eye movements in reading.

We have shown that binocular FD may be seen as integral to normal reading, with specific behaviors that are interpretable as adaptive. This theory of binocularity presents a challenge to researchers modeling eye movements in reading, either to accommodate binocularity into implemented models of reading or to show that binocular data are lost to the reading system relatively peripherally and, therefore, become irrelevant to the central execution of reading.

\section{Conclusions}

Solving the correspondence problem-achieving fusion - is of prime importance in reading, as it is in other binocular tasks. The correspondence problem elicited by binocular FDs in reading resembles aspects of the correspondence problem found for the images of objects in front of or beyond the horopter: The two images are offset in one or other direction. Crossed RDs in depth perception resemble uncrossed FDs in reading, and uncrossed RDs in depth perception resemble crossed FDs in reading.

The fusion-related processing of crossed RDs is more effective and robust than is the comparable processing of uncrossed RDs, in development, in impairment, and over a range of behaviors elicited in experiments on normal adult participants. Uncrossed FDs are, therefore, expected to be the default direction of FD when the stimulus configuration is perceptually challenging and stereofusion is problematic.

When the overall stimulus configuration is not perceptually challenging and stereofusion is assured, then more effective non-fusion-related processing can be achieved by making maximal use of the nasal hemifoveas - as occurs in a crossed FD-reflecting foveal splitting and the contralateral preference.

Binocular FD in reading allows a coherent extension of the split-fovea model of reading. It does not militate 
against the split-fovea theory or make the theory irrelevant to reading, as some researchers have recently suggested.

This theory currently offers the only explanation for the emerging data on binocularity derived from eyetracking and dichoptic presentations. Such data challenge all current models of reading, opening exciting theoretical possibilities.

\section{AUTHOR NOTE}

This work was supported by Project Grants R39195 and R39942 from the ESRC (U.K.). The present article reports partial analyses of corpus data collected under Project Grant R39195. We thank Graeme Phillipson for advice on depth perception and for producing Figure 3. With regard to the Edinburgh Five-Language Corpus, we acknowledge Scott McDonald for his leading role in the corpus design and collection, for the development of analysis software, and for his insightful discussions. We also thank Ralph Radach, Hazel Blythe, Wolfgang Jaschinski, the editor, and an anonymous reviewer for helpful comments. Any remaining errors are due to the authors. Address correspondence to R. Shillcock, Department of Psychology, University of Edinburgh, 7 George Square, Edinburgh, EH8 9JZ Scotland (e-mail: rcs@inf.ed.ac.uk).

\section{REFERENCES}

Alais, D., \& Blake, R. (2005). Binocular rivalry. Cambridge, MA: MIT Press.

Anstis, S. M. (1974). A chart demonstrating variations in acuity with retinal position. Vision Research, 14, 589-592.

BACH, M., Bouis, D., \& Fischer, B. (1983). An accurate and linear infrared oculometer. Journal of Neuroscience Methods, 9, 9-14.

Bassou, L., Granié, M., Pugh, A. K., \& Morucci, J. P. (1992). La coordination binoculaire pendant la lecture. Comptes Rendus de l'Academie des Sciences: Serie III, Sciences die la vie, 315, 159-164.

Beard, B. L., Levi, D. M., \& Klein, S. A. (1997). Vernier acuity with non-simultaneous targets: The cortical magnification factor estimated by psychophysics. Vision Research, 37, 325-346. doi:10.1016/S0042 -6989(96)00109-5

Beverley, K. I., \& Regan, D. (1974). Visual sensitivity to disparity pulses: Evidence for directional selectivity. Vision Research, 14, 357361. doi:10.1016/0042-6989(74)90095-9

Birch, E. E., \& Foley, J. M. (1979). The effects of duration and luminance on binocular depth mixture. Perception, 8, 263-267. doi:10.1068/p080263

Birch, E. E., Gwiazda, J., \& Held, R. (1982). Stereoacuity development for crossed and uncrossed disparities in human infants. Vision Research, 22, 507-513. doi:10.1016/0042-6989(82)90108-0

BlaKemore, C. (1969). Binocular depth discrimination and the nasotemporal division. Journal of Physiology, 205, 471-497.

Blythe, H. I., Liversedge, S. P., Joseph, H. S., White, S. J., FindLAY, J. M., \& RAYNER, K. (2006). The binocular coordination of eye movements during reading in children and adults. Vision Research, 46, 3898-3908.

Bruce, V., Green, P. R., \& Georgeson, M. A. (1996). Visual perception: Physiology, psychology, and ecology (3rd ed.). Hove, U.K.: Psychology Press.

BRYSBAERT, M. (1994). Interhemispheric transfer and the processing of foveally presented stimuli. Behavioural Brain Research, 64, 151-161.

BRysBaERT, M. (2004). The importance of interhemispheric transfer for foveal vision: A factor that has been overlooked in theories of visual word recognition and object perception. Brain \& Language, 88, 259267.

CнAско, L. W. (1948). The laminar pattern of the lateral geniculate body in the primates. Journal of Neurology, Neurosurgery, \& Psychiatry, 11, 211-224.

Clark, B. (1935). The effect of binocular imbalance on the behavior of the eyes during reading. Journal of Educational Psychology, 26, $530-538$.

Collewijn, H., Erkelens, C. J., \& Steinman, R. M. (1988). Binocular co-ordination of human horizontal saccadic eye movements. Journal of Physiology, 404, 157-182.
Collewijn, H., Erkelens, C. J., \& Steinman, R. M. (1995). Voluntary binocular gaze-shifts in the plane of regard: Dynamics of version and vergence. Vision Research, 35, 3335-3358.

Collewijn, H., Steinman, R. M., Erkelens, C. J., \& Regan, D. (1991). Binocular fusion, stereopsis, and stereoacuity with a moving head. In D. Regan (Ed.), Vision and visual dysfunction: Vol. 9. Binocular vision (pp. 121-136). New York: Macmillan.

Conner, I. P., Odom, J. V., Schwartz, T. L., \& Mendola, J. D. (2007). Retinotopic maps and foveal suppression in the visual cortex of amblyopic adults. Journal of Physiology, 583, 159-173.

Cornell, E. D., Macdougall, H. G., Predebon, J., \& Curthoys, I. S. (2003). Errors of binocular fixation are common in normal subjects during natural conditions. Optometry \& Vision Science, 80, 764-771.

Cumming, B. G., \& PARKer, A. J. (1999). Binocular neurons in V1 of awake monkeys are selective for absolute, not relative, disparity. Journal of Neuroscience, 19, 5602-5618.

DEAnGelis, G. C. (2000). Seeing in three dimensions: The neurophysiology of stereopsis. Trends in Cognitive Sciences, 4, 80-90. doi:10.1016/ S1364-6613(99)01443-6

Dengler, B., \& Kommerell, G. (1993). Stereoscopic cooperation between the fovea of one eye and the periphery of the other eye at large disparities: Implications for anomalous retinal correspondence in strabismus. Graefe's Archive for Clinical \& Experimental Ophthalmology, 231, 199-206. doi:10.1007/BF00918841

Ehrenstein, W. H., Arnold-Schulz-Gahmen, B. E., \& JaschinSKI, W. (2005). Eye preference within the context of binocular functions. Graefe's Archive for Clinical \& Experimental Ophthalmology, 243, 926-932.

ElLIS, A. W. (2004). Length, formats, neighbours, hemispheres, and the processing of words presented laterally or at fixation. Brain \& Language, 88, 355-366.

Ellis, A. W., \& Brysbaert, M. (2010). Split fovea theory and the role of the two cerebral hemispheres in reading: A review of the evidence. Neuropsychologia , 48, 353-365. doi:10.1016/j.neuropsychologia .2009.08.021

ENRIGHT, J. T. (1998). Monocularly programmed human saccades during vergence changes. Journal of Physiology, 512, 235-258.

Erkelens, C. J., MuiJs, A. J. M., \& van Ee, R. (1996). Binocular alignment in different depth planes. Vision Research, 36, 2141-2147.

Erkelens, C. J., Steinman, R. M., \& Collewijn, H. (1989). Ocular vergence under natural conditions: II. Gaze shifts between real targets differing in distance and direction. Proceedings of the Royal Society $B, \mathbf{2 3 6}, 441-465$.

Fioravanti, F., Inchingolo, P., Pensiero, S., \& Spanio, M. (1995). Saccadic eye movement conjugation in children. Vision Research, 35, 3217-3228.

Foley, J. M. (1976). Binocular depth mixture. Vision Research, 16, 1263-1267.

Foley, J. M. (1980). Binocular distance perception. Psychological Review, 87, 411-434.

Foley, J. M., \& Richards, W. A. (1978). Binocular depth mixture with non-symmetric disparities. Vision Research, 18, 251-256. doi:10.1016/0042-6989(78)90159-1

Godek, C. L., \& Lawson, R. B. (1973). The effects of stimulus duration, time interval between stimuli, and disparity upon stereoscopic depth perception. Psychological Record, 23, 243-248.

Goutcher, R., \& Mamassian, P. (2006). Temporal dynamics of stereo correspondence bi-stability. Vision Research, 46, 3575-3585.

GrabowsKa, A. (1983). Lateral differences in the detection of stereoscopic depth. Neuropsychologia, 21, 249-257. doi:10.1016/0028 -3932(83)90041-6

HaAse, H.-J. (1995). Zur Fixationsdisparation. Heidelberg: Optische Fachveröffentlichung GmbH.

Heinrich, S. P., Kromeier, M., Bach, M., \& Kommerell, G. (2005). Vernier acuity for stereodisparate objects and ocular prevalence. $\mathrm{Vi}$ sion Research, 45, 1321-1328.

Held, R., Birch, E., \& Gwiazda, J. (1980). Stereoacuity of human infants. Proceedings of the National Academy of Sciences, 77, 55725574.

Heller, D., \& RADACH, R. (1999). Eye movements in reading: Are two eyes better than one? In W. Becker, H. Deubel, \& T. Mergner (Eds.), Current oculomotor research: Physiological and psychological aspects (pp. 341-348). New York: Plenum. 
Henderson, J. M., \& Ferreira, F. (1990). Effects of foveal processing difficulty on the perceptual span in reading: Implications for attention and eye movement control. Journal of Experimental Psychology: Learning, Memory, \& Cognition, 16, 417-429.

HendRIKs, A. W. (1992). Vergence eye movements as a function of text type and of reading objective [Abstract]. Perception, 21, 51.

HENDRIKS, A. W. (1996). Vergence eye movements during fixations in reading. Acta Psychologica, 92, 131-151.

Hendriks, A. W., Kolk, H. H. J., \& VAN DER Wildt, G. J. (1991). Vergence eye movements as a function of text type and of reading objective [Abstract]. In J. Van Rensbergen, M. Devijver, \& G. d'Ydewalle (Eds.), Proceedings of the Sixth European Conference on Eye Movements (Add.) (pp. 1-3). Leuven: University of Leuven, Faculty of Psychology and Educational Sciences.

Hershenson, M. (1999). Visual space perception. Cambridge, MA: MIT Press.

Hillemanns, M. (1927). Die funktionelle Asymmetrie der Augen, die Vorherrschaft eines derselben und die binokulare Richtungslokalization. Klinische Monatsblätter für Augenheilkunde, 78, 737-761.

HorTON, J. C., \& HockING, D. R. (1996). Intrinsic variability of ocular dominance column periodicity in normal macaque monkeys. Journal of Neuroscience, 16, 7228-7239.

HowARD, I. P., \& ROGERS, B. J. (1995). Binocular vision and stereopsis. New York: Oxford University Press.

Howard, I. P., \& Rogers, B. J. (2002). Seeing in depth: Vol. 2. Depth perception. Toronto: Porteous.

JACOBS, R. J. (1979). Visual resolution and contour interaction in the fovea and periphery. Vision Research, 19, 1187-1196.

JAINTA, S., \& JASCHINSKI, W. (2002). Fixation disparity: Binocular vergence accuracy for a visual display at different positions relative to the eyes. Human Factors, 44, 443-450.

JASCHINSKI, W. (1997). Fixation disparity and accommodation as a function of viewing distance and prism load. Ophthalmic \& Physiological Optics, 17, 324-339.

JASCHINSKI, W. (2001). Fixation disparity and accommodation for stimuli closer and more distant than oculomotor tonic positions. Vision Research, 41, 923-933.

JASCHINSKI, W. (2002). The proximity-fixation-disparity curve and the preferred viewing distance at a visual display as an indicator of near vision fatigue. Optometry \& Vision Science, 79, 158-169.

JASCHINSKI, W., BRÖDE, P., \& GRIEFAHN, B. (1999). Fixation disparity and nonius bias. Vision Research, 39, 669-677.

JaschinSKI, W., KoItcheVA, V., \& Heuer, H. (1998). Fixation disparity, accommodation, dark vergence, and dark focus during inclined gaze. Ophthalmic \& Physiological Optics, 18, 351-359. doi:10.1016/ S0275-5408(98)00011-8

JASCHINSKI, W., \& SCHROTh, V. (2008). Ocular prevalence: Difference between crossed and uncrossed disparities of stereo objects. Strabismus, 16, 159-164. doi:10.1080/09273970802451059

JASCHINSKI-KRUZA, W. (1994). Dark vergence in relation to fixation disparity at different luminance and blur levels. Vision Research, 34, 1197-1204. doi:10.1016/0042-6989(94)90301-8

Jenkins, T. C. A., Pickwell, L. D., \& Yekta, A. A. (1989). Criteria for decompensation in binocular vision. Ophthalmic \& Physiological Optics, 9, 121-125

JoRDAN, T. R., \& PATERSON, K. B. (2009). Re-evaluating split-fovea processing in word recognition: A critical assessment of recent research. Neuropsychologia, 47, 2341-2353.

Juhasz, B. J., Liversedge, S. P., White, S. J., \& Rayner, K. (2006). Binocular coordination of the eyes during reading: Word frequency and case alternation affect fixation duration but not fixation disparity. Quarterly Journal of Experimental Psychology, 59, 1614-1625.

JULESZ, B. (1964). Binocular depth perception without familiarity cues. Science, 145, 356-362.

Kirkby, J. A., Webster, L. A., Blythe, H. I., \& Liversedge, S. P. (2008). Binocular coordination during reading and non-reading tasks. Psychological Bulletin, 134, 742-763.

Kliegl, R., Grabner, E., Rolfs, M., \& Engbert, R. (2004). Length, frequency and predictability effects of words on eye movements in reading. European Journal of Cognitive Psychology, 16, 262-284.

Kommerell, G., Schmitt, C., Kromeier, M., \& Bach, M. (2003).
Ocular prevalence versus ocular dominance. Vision Research, 43, 1397-1403.

Kupfer, C., Chumbley, L., \& Downer, J. C. (1967). Quantitative histology of optic nerve, optic tract, and lateral geniculate nucleus of man. Journal of Anatomy, 101, 393-401.

Lam, A. K. C., Tse, P., Choy, E., \& Chung, M. (2002). Crossed and uncrossed stereoacuity at distance and the effect from heterophoria. Ophthalmic \& Physiological Optics, 22, 189-193.

Lasley, D. J., Kivlin, J., Rich, L., \& Flynn, J. T. (1982, March). Diplopic stereo discrimination. Paper presented at the meeting of the Association for Research in Vision and Ophthalmology, Sarasota, FL.

LAVIDOR, M., \& WALSH, V. (2004). The nature of foveal representation. Nature Reviews Neuroscience, 5, 729-735.

LEFF, A. P. (2004). A historical review of the representation of the visual field in primary visual cortex with special reference to the neural mechanisms underlying macular sparing. Brain \& Language, 88, 268-278.

LeVay, S., Connolly, M., Houde, J., \& Van Essen, D. C. (1985). The complete pattern of ocular dominance stripes in the striate cortex and visual field of the macaque monkey. Journal of Neuroscience, 5, 486501.

Levi, D. M., Klein, S. A., \& Aitsebaomo, A. P. (1985). Vernier acuity, crowding and cortical magnification. Vision Research, 25, 963-977.

Liversedge, S. P., \& Blythe, H. I. (2009, April). There's no such thing as a free trip across the corpus callosum. Talk presented at the Leicester meeting of the Experimental Psychology Society.

Liversedge, S. P., White, S. J., Findlay, J. M., \& Rayner, K. (2006). Binocular coordination of eye movements during reading. Vision Research, 46, 2363-2374.

Malinov, I. V., Epelboim, J., Herst, A. N., \& Steinman, R. M. (2000). Characteristics of saccades and vergence in two kinds of sequential looking tasks. Vision Research, 40, 2083-2090.

McDonald, S. A., \& Shillcock, R. C. (2005a). The implications of foveal splitting for saccade planning in reading. Vision Research, $\mathbf{4 5}$ 801-820. doi:10.1016/j.visres.2004.10.002

McDonald, S. A., \& SHILlcock, R. C. (2005b). Toward an appropriate baseline for measures of eye movement behavior during reading. Journal of Experimental Psychology: Human Perception \& Performance, 31, 584-591. doi:10.1037/0096-1523.31.3.584

Monaghan, P., \& Shillcock, R. C. (2008). Hemispheric dissociation and dyslexia in a computational model of reading. Brain \& Language, 107, 185-193. doi:10.1016/j.bandl.2007.12.005

Monaghan, P., Shillcock, R., \& McDonald, S. (2004). Hemispheric asymmetries in the split-fovea model of semantic processing. Brain \& Language, 88, 339-354. doi:10.1016/S0093-934X(03)00165-2

MustilLo, P. (1985). Binocular mechanisms mediating crossed and uncrossed stereopsis. Psychological Bulletin, 97, 187-201.

Nuthmann, A., \& Kliegl, R. (2009). An examination of binocular reading fixations based on sentence corpus data. Journal of Vision, 9(5, Art. 31) 1-28.

Obregón, M., \& Shillcock, R. (2007, August). A contralateral hemifoveal advantage in isolated word recognition. Talk presented at ECEM2007, 14th European Conference on Eye Movements, Potsdam. Abstract retrieved from http://users.utu.f1/latanu/PDFs/bastracts_ecem14.pdf.

Obregón, M., \& Shillcock, R. (2010). An advantage for the contralateral over ipsilateral visual pathways in foveal word identification. Manuscript in preparation.

Ogle, K. N. (1963). Stereoscopic depth perception and exposure delay between images to the two eyes. Journal of the Optical Society of America, 53, 1296-1304. doi:10.1364/JOSA.53.001296

O'Regan, J. K., LÉvy-Schoen, A., \& Jacobs, A. M. (1983). The effect of visibility on eye-movement parameters in reading. Perception \& Psychophysics, 34, 457-464.

Perry, V. H., \& Cowey, A. (1985). The ganglion cell and cone distributions in the monkey's retina: Implications for central magnification factors. Vision Research, 25, 1795-1810. doi:10.1016/0042 $-6989(85) 90004-5$

PICKWEll, D. (1989). Binocular vision anomalies: Investigation and treatment. London: Butterworth-Heinemann.

Pickwell, D. (1991, March 25). The binocular stress syndromes. Optometry Today, p. 6. 
Pickwell, D., Jenkins, T., \& Yekta, A. A. (1987). The effect of fixation disparity and associated heterophoria of reading at an abnormally close distance. Ophthalmic \& Physiological Optics, 7, 345-347.

Poggio, G. F., \& Fischer, B. (1977). Binocular interaction and depth sensitivity in striate and prestriate cortex of behaving rhesus monkey. Journal of Neurophysiology, 40, 1392-1405.

Radach, R., Huestegge, L., \& Reilly, R. (2008). The role of global top-down factors in local eye-movement control in reading. Psychological Research, 72, 675-688. doi:10.1007/s00426-008-0173-3

Regan, D., \& Beverley, K. I. (1973a). Disparity detectors in human depth perception: Evidence for directional selectivity. Science, 181, 877-879.

Regan, D., \& Beverley, K. I. (1973b). Some dynamic features of depth perception. Vision Research, 13, 2369-2379.

REgan, D., \& SPEKREIJSE, H. (1970). Electrophysiological correlate of binocular depth perception in man. Nature, 255, 92-94.

Reinhard, J., \& TrauZettel-Klosinski, S. (2003). Nasotemporal overlap of retinal ganglion cells in humans: A functional study. Investigative Ophthalmology \& Visual Science, 4, 1568-1572. doi:10.1167/ iovs.02-0313

RICHARDS, W. A. (1970). Stereopsis and stereoblindness. Experimental Brain Research, 10, 380-388.

Richards, W. A. (1971a). Anomalous stereoscopic depth perception. Journal of the Optical Society of America, 61, 410-419.

RicHARDS, W. A. (1971b). Independence of Panum's near and far limits. American Journal of Optometry \& Archives of the American Academy of Optometry, 48, 103-109.

RICHARDS, W. A. (1973). Reversal in stereo discrimination by contrast reversal. American Journal of Optometry \& Archives of the American Academy of Optometry, 50, 853-862.

RichaRds, W. A., \& Foley, J. M. (1971). Interhemispheric processing of binocular disparity. Journal of the Optical Society of America, 61, 419-421.

Richards, W. A., \& Regan, D. (1973). A stereo field map with implications for disparity processing. Investigative Ophthalmology, 12, 904-909.

Roberts, M. A. J., Kreiner, H., Obregón, M., \& Shillcock, R. C. (2009). Binocular strategies in reading. Talk presented at ECEM '09. Southampton, U.K.

Roberts, M. A. J., Shillcock, R. C., Kreiner, H., \& Obregón, M. (2010). Wider fixation disparity is followed by longer saccades in reading. Manuscript submitted for publication.

Rombouts, S. A., Barkhof, F., Sprenger, M., Valk, J., \& SchelTENS, P. (1996). The functional basis of ocular dominance: Functional MRI (fMRI) findings [published erratum appears in Neuroscience Letters, 1997, March 14, 224, 147]. Neuroscience Letters, 221, 1-4.

Sachsenweger, R. (1958). Sensorische Fusion und Schielen. Graefe's Archive for Clinical \& Experimental Ophthalmology, 159, 502528.

SCHLOSBERG, H. (1941). Stereoscopic depth from single pictures. American Journal of Psychology, 54, 601-605. doi:10.2307/1417214

SCHMidT, W. A. (1917). An experimental study in the psychology of reading. A dissertation. Supplementary Educational Monographs, 1(2). Chicago: University of Chicago Press.

Schor, C. M., \& Wood, I. (1983). Disparity range for local stereopsis as a function of luminance spatial-frequency. Vision Research, 23, 1649-1654. doi:10.1016/0042-6989(83)90179-7

Setter, P., \& Norman, J. (2006). A study of human stereoscopic eyemovements to provide data for stereo computer vision models (Report). University of Haifa: Caesarea-Rothschild Foundation.

Shillcock, R., Ellison, T. M., \& Monaghan, P. (2000). Eye-fixation behavior, lexical storage, and visual word recognition in a split processing model. Psychological Review, 107, 824-851.

Shillcock, R., Roberts, M. A. J., Kreiner, H., \& Mac Cumhaill, C. (2007, August). Crossed and uncrossed fixation disparities in reading; eye-tracking, technologies, individual differences and illumination. Poster presented at ECEM2007, 14th European Conference on Eye Movements Potsdam. Abstract retrieved from http://users.utu.f1/ latanu/PDFs/bastracts_ecem14.pdf.

TAYLOR, E. A. (1966). The fundamental reading skill, as related to eye movement photography and visual anomalies (2nd ed.). Springfield, IL: Charles C. Thomas.

Toosy, A. T., Werring, D. J., Plant, G. T., Bullmore, E. T., Miller,
D. H., \& Thompson, A. J. (2001). Asymmetrical activation of human visual cortex demonstrated by functional MRI with monocular stimulation. NeuroImage, 14, 632-641. doi:10.1006/nimg.2001.0851

TyLeR, C. W. (1991). Cyclopean vision. In D. Regan (Ed.), Vision and visual disorders: Vol. 9. Binocular vision (pp. 38-74). New York: Macmillan.

Tyler, C. W. (2004). Binocular vision. In W. Tasman, \& E. A. Jaeger (Eds.), Duane's foundations of clinical ophthalmology: Vol. 2 (pp. 1-29). Philadelphia: Lippincott.

Woo, G. G. S., \& SillanpaA, V. (1979). Absolute stereoscopic thresholds as measured by crossed and uncrossed disparities. American Journal of Optometry \& Physiological Optics, 56, 350-355.

YANG, Q., \& KAPOULA, Z. (2003). Binocular coordination of saccades at far and at near in children and in adults. Journal of Vision, 3, 554561.

Yen, M.-H., \& RADACH, R. (2007, August). Saccades and binocular coordination in reading: Effects of viewing distance and font size. Talk presented at ECEM2007, 14th European Conference on Eye Movements, Potsdam. Abstract retrieved from http://users.utu.fl/latanu/ PDFs/bastracts_ecem14.pdf.

YGGE, J., \& JACOBSON, C. (1994). Asymmetrical saccades in reading. In J. Ygge \& G. Lennerstrand (Eds.), Eye movements in reading (pp. 301313). Oxford: Pergamon.

Zaroff, C. M., Knutelska, M., \& Frumkes, T. E. (2003). Variation in stereoacuity: Normative description, fixation disparity, and the roles of aging and gender. Investigative Ophthalmology \& Visual Science, 44, 891-900. doi:10.1167/iovs.02-0361

\section{NOTES}

1. We use the term disconjugacy only when we also consider the saccade.

2. We thank a reviewer for pointing up this issue. The precise implications of binocular versus monocular calibration are unknown and likely to be complex, but we suggest that binocular calibration is conservative, overall, with respect to binocular FD, in that a possible binocular FD is effectively being "zeroed" by a binocular calibration, so that any subsequent nonzero FDs of the same sign underestimate the objective disparity.

3. There are two aspects to this difference between sentential and paragraph materials. The first is the degree to which the fixation stimulus at the beginning of a trial requires a precisely conjoint fixation and how frequently this requirement is reinforced during the experiment. The second is less easily specified, but Radach, Huestegge, and Reilly (2008) have demonstrated the importance of this difference in materials for monocular eye movements. Heller and Radach (1999) reported that disparity increases during the reading of a line, as did Nuthmann and Kliegl (2009). This effect is also present in our own corpus data, as is an increase in disparity over successive lines of text in the same paragraph.

4. Angle subtended by each letter is just one of the variables suggested by Nuthmann and Kliegl (2009); we have selected this variable simply as a convenient proxy for legibility. We will go on to claim that uncrossed FDs in reading are prompted by conditions that make binocular fusion difficult.

5. The optometric prefixes eso- ("in") and exo- ("out") may also be used, respectively, to refer to the overconverged and underconverged states being referred to here as "crossed" and "uncrossed" binocular fixations on the text

6. Although we have used the standard term RD in our discussion of the stereopsis literature, FDs naturally have retinal implications; indeed, the key point we will make is that we can understand the retinal implications of FDs in reading in terms of the processing of RDs in depth perception.

7. Indeed, in real-world fixation behavior, we may extend this description to say that noncorrespondence between the retinal images caused by the structure of the visual scene in depth and by nonconjugate saccadic movements and fixations is also typically copresent.

8. Albeit with some ranking, that means that contour will typically be more influential in binocular fusion in any conflict with accommodation and vergence information (see, e.g., Bruce, Green, \& Georgeson, 1996, p. 138; Foley, 1980; see also Alais \& Blake, 2005).

9. We will not pursue the suggestion, briefly considered by Mustillo (1985), that conjoint fixations may access a third processing domain. 
10. N.B.-Our claim is not that there is perceived depth in normal reading; we clearly do not see depth comparable to that elicited by haploscope-induced disparity, for instance. However, the absence of any phenomenological depth in the experience of reading is a moot point; anecdotally, the so-called "plastic effect" (see, e.g., Schlosberg, 1941) does seem to obtain in reading, for instance.

11. Data available on request, along with that from six normal readers who showed no effect of room illumination.

12. We have made the distinction between fusion-related processing (facilitated by uncrossed FDs) and postfusional processing (facilitated by crossed FDs), but the latter term reflects our interest in the eventual reading behaviors and might also have been termed "processing not directly connected with fusion," given that it includes such factors as the projection of visual information from the retina.

13. Data available on request.

14. One reviewer has suggested that larger FDs should be predictive of shorter fixation times in normal reading. We do not believe that such a simple prediction will be confirmed and have found no evidence in our data that it is true; Liversedge and Blythe (2009) have reported the absence of such an effect in their own data. We suggest that FDs of different sizes and signs correlate with different styles of hemispheric processing. We have said that greater representational activation should facilitate postfusional processing, ceteris paribus. Our prediction is that what we have called representational activation will have more effect on lower level perceptual processing than on the higher level cognition that is reflected in fixation durations. Extending the window onto the text (by widening FD) might involve similarly spreading out the constant processing resources more thinly (cf. Henderson \& Ferreira, 1990), so that any advantages of a wider window onto the text are offset by having more work to do with the same resources.

(Manuscript received April 7, 2009; revision accepted for publication June 21, 2010.) 